\title{
Gas of wormholes: a possible ground state of Quantum Gravity
}

\author{
G. Preparata, S. Rovelli, S.-S. Xue ${ }^{(a)}$ \\ Dipartimento di Fisica dell'Università and INFN - sezione di Milano, Via \\ Celoria 16, Milan, Italy \\ (a) I.C.R.A. - International Center of Relativistic Astrophysics and INFN, La \\ Sapienza, 00185 Rome, Italy.
}

\begin{abstract}
In order to gain insight into the possible Ground State of Quantized Einstein's Gravity, we have derived a variational calculation of the energy of the quantum gravitational field in an open space, as measured by an asymptotic observer living in an asymptotically flat space-time. We find that for Quantum Gravity (QG) it is energetically favourable to perform its quantum fluctuations not upon flat space-time but around a "gas" of wormholes of mass $m_{p}$, the Planck mass $\left(m_{p} \simeq 10^{19} \mathrm{GeV}\right)$ and average distance $l_{p}$, the Planck length $a_{p}\left(a_{p} \simeq 10^{-33} \mathrm{~cm}\right)$. As a result, assuming such configuration to be a good approximation to the true Ground State of Quantum Gravity, space-time, the arena of physical reality, turns out to be well described by Wheeler's quantum foam and adequately modeled by a space-time lattice with lattice constant $l_{p}$, the Planck lattice.
\end{abstract}

PACS $04.60, \ldots$ (other pacs) 


\section{Introduction}

Among the fundamental interactions of Nature, since the monumental contribution of Albert Einstein, Gravity plays the central role of determining the structure of space-time, the arena of physical reality. As well known, in classical physics a world without matter, the Vacuum, has the simplest of all structures, it is flat (pseudoeuclidean); but in quantum physics? This is the focal question that has occupied the best theoretical minds since it became apparent, at the beginning of the 30's, that Quantum Field Theory (QFT') is the indispensable intellectual tool for discovering the extremely subtle ways in which the quantum world actually works. Thus the problem to solve was to find in some way or other the Ground State (GS) of Quantum Gravity (QG), which determines the dynamical behaviour of any physical system, through the non-trivial structure that space-time acquires as a result of the quantum fluctuations that in such state the gravitational field, like all quantum fields, must experience. Of course this problem, at least in the non-perturbative regime, is a formidable one, and many physicists, J.A. Wheeler foremost among them, could but speculate about the ways in which the expected violent quantum fluctuations at the Planck distance $l_{p}\left(l_{p}=10^{-33} \mathrm{~cm}\right)$ could change the space-time structure of the Vacuum, from its classical, trivial (pseudoeuclidean) one. And Wheeler's conjecture, most imaginative and intriguing, of a space-time foam vividly expressed the intuition that at the Planck distance the fluctuations of the true QG ground state would end up in submitting the classical continuum of events to a metamorphosis into an essentially discontinuous, discrete structure

It is the purpose of this paper to give a detailed account of the results of an investigation on a possible QG ground state, which has been summarily reported in a recent letter [1]. The starting point of our attack is the realization that QG can be looked at as a non-abelian gauge theory whose gauge group is the Poincaré group. Following the analysis performed by one of us (GP) [2] of another nonabelian gauge theory QCD (whose gauge group is $S U_{c}(3)$ ), we decided to explore the possibility that the energy density (to be appropriately defined, see below) of the quantum fluctuations of the gravitational field around a non-trivial classical solution of the Einstein's field equations for the matterless world, could be lower than the energy of the perturbative ground state (PGS), which comprises the zero point fluctuations of the gravitational field's modes around flat space-time. Indeed in QCD it was found that the unstable modes (imaginary frequencies) of the gauge fields around the classical constant chromomagnetic field solution of the empty space Yang-Mills equations, in the average screen completely the classical chromomagnetic field, allowing the interaction energy between such field

\footnotetext{
${ }^{1}$ We should like to recall here that, based on Wheeler's idea, a successful research program was initiated a few years ago to explore the consequences of the Standard Model $\left(S U_{c}(3) \otimes\right.$ $\left.S U_{L}(2) \otimes U_{Y}(1)\right)$ in a discrete space-time, conveniently modeled by a lattice of constant $l_{p}$, the Planck lattice (PL).
} 
and the short wave-length fluctuations of the quantized gauge field, to lower the energy density of such configuration below the PGS energy density. Thus we decided to try for QG the strategy that was successful in QCD, i.e.

1. select a class of empty space classical solutions of Einstein's equations that is simple and manageable;

2. evaluate the spectrum of the small amplitude fluctuations of the gravitational field around such solutions;

3. set up a variational calculation of the appropriately defined energy density in the selected background fields;

4. study the possible screening by the unstable modes (if any) of the classical background fields.

As for point (1) we have chosen the Schwarzschild's wormhole-solutions [3], the simplest class of solutions of Einstein's equation after flat space-time. In order to achieve (2) the Regge-Wheeler [- 1 expansion has been systematically employed, yielding a well defined set of unstable modes (for S-wave). This important result, already indicated in previous independent work [5], renders the development of the points (3) and (4) both relevant and meaningful, the former point yielding a lowering of the energy density due to the interaction of the short-wave length modes with the background gravitational field, the latter exhibiting the (approximate) cancellation of the independent components of the tensor of the Schwarzschild's wormholes by the S-wave unstable modes. As a result flat spacetime, like the QCD perturbative ground state, becomes "essentially unstable", in the sense that upon it no stable quantum dynamics can be realized. On the other hand a well defined "gas" of wormholes appears as a very good candidate for the classical configuration around which the quantized modes of the gravitational field can stably fluctuate. But a discussion of the physical implications of our findings must await a more detailed description of our work, which we are now going to provide.

\section{The Schrödinger functional approach}

In order to develop a functional strategy aimed at determining the Ground State of Quantum Gravity, which parallels the approach developed for QCD [2], we must first identify an appropriate energy functional. In General Relativity this is a non-trivial problem for, as is well known, in the canonical quantization procedure, first envisaged by Dirac [6] and Arnowitt, Deser and Misner (ADM) [7], due to general covariance the local Hamiltonian is constrained to annihilate the physical ground state, a fact that in the Schrödinger functional approach is expressed by the celebrated Wheeler-DeWitt equation [8]. However we note that 
the problem we wish to solve concerns the minimization of the total energy of an "open space", in which there exists a background metric field that becomes "asymptotically flat", i.e. that for spatial infinity $(r=|\vec{x}| \rightarrow \infty)$ behaves as

$$
g_{\mu \nu} \longrightarrow \eta_{\mu \nu}^{(M)}+O\left(\frac{1}{r}\right)
$$

where $\eta_{\mu \nu}^{(M)}$ is the Minkowski metric. In the conventional canonical formulation, space-time is foliated into spacelike slices $\Sigma$ with constant values of the time parameter $t$; the asymptotic condition (1) determines the asymptotic behaviour of the canonical variables: the spatial 3-metric $g_{i j}$ on $\Sigma$, the conjugate momenta $\pi^{i j}$, the "lapse-function" $N$ and the "shift- vector" $N_{i}$ [7] as:

$$
\begin{aligned}
g_{i j} & \longrightarrow \delta_{i j}+O\left(r^{-1}\right) \\
\pi^{i j} & \longrightarrow O\left(r^{-2}\right) \\
N & \longrightarrow 1 \\
N_{i} & \longrightarrow 0 .
\end{aligned}
$$

Let us consider the ADM-energy [7], which in cartesian coordinates is given by ( $\partial \Sigma$ is the boundary of $\Sigma$, ", $k$ " denotes partial derivative with respect to $x_{k}$, and $G=l_{p}^{2}$ is the Newton constant)

$$
E_{A D M}=\frac{1}{16 \pi G} \int_{\partial \Sigma} d S^{k} \delta^{i j}\left(g_{i k, j}-g_{i j, k}\right) .
$$

We should like to point out that $E_{A D M}$ is just the energy that an asymptotic observer attributes to a space region $\Sigma$ whose time foliation he is keeping anchored to his (asymptotically) flat metric: the boundary conditions select a privileged reference frame (up to Lorentz transformations) that implicitly defines the physically relevant energy. In particular, the asymptotic condition on $N$ fixes the "boundary time" unequivocally: the asymptotic observer is the only possessor of an idealized clock that allows him to describe quantities associated to the whole physical system without introducing material clocks (i.e. auxiliary fields). In this sense, he is also the only one that can really be termed as an idealized, non-interfering "observer" capable of describing geometry at the quantum level in terms of evolution, not merely in terms of correlation between variables, thus giving a full meaning to the expression "quantum geometrodynamics" 9].

At the classical level, the definition of $E_{A D M}$ fixes Minkowski geometry as the zero point of the total energy; the proof of its positivity [10] can be looked at as the statement that flat space-time is the (unique) vacuum of General Relativity. This explains why the first steps towards the quantization of the theory have been based on a perturbative approach on the flat background, with the fluctuating self-interacting field interpreted in the conventional particle view as creating and annihilating gravitons, which propagate in pseudo-euclidean space: in this sense, 
we call flat space-time filled with gravitons performing zero-point fluctuations the "perturbative ground state" (PGS).

We now know that the perturbative approach was doomed to fail: the nonrenormalizability of the theory does not allow to make any meaningful and predictive perturbative expansion. On the other hand, analyzing the theory to the lowest non-trivial order around a curved background may give us important indications of how the deadly "impasse" of the perturbative approach may be finally overcome and give back to the simplest form of QG its status and role of a "bona fide" Quantum Field Theory.

We thus study the quantum fluctuations of the gravitational field upon a generic, asymptotically flat stationary background geometry, solution of the sourceless Einstein's equations; in particular, for the background metric we can choose a foliation orthogonal to Killing timelike vectors and put it in static form. On a given slice $\Sigma$, the 3 -metric is thus given by

$$
g_{i j}(x)=\eta_{i j}(x)+h_{i j}(x),
$$

where $\eta_{i j}(x)$ is the spatial background metric and $h_{i j}(x)$ the fluctuation to be quantized $(x \in \Sigma)$. We can now expand the total ( the sum of the Hamiltonian and $E_{A D M}$ ) energy $E$ of space in powers of the fluctuations $h_{i j}(x)$ (the number (n) denotes the order of the expansion):

$$
E=E_{A D M}^{(0)}+E_{A D M}^{(1)}+\sum_{n \geq 1} \int_{\Sigma} d^{3} x\left(N \mathcal{H}^{(n)}+N_{i} \mathcal{H}^{i(n)}\right)
$$

$\mathcal{H}$ and $\mathcal{H}^{i}$ are the super-hamiltonian and super- momentum, as defined by ADM [7]:

$$
\begin{aligned}
\mathcal{H} & \equiv T+V \\
T & =16 \pi G \mathcal{G}_{i j k l} \pi^{i j} \pi^{k l} \\
V & =-\frac{g^{1 / 2}}{16 \pi G} R
\end{aligned}
$$

where $g$ is the determinant of 3-metric and $R$ the corresponding curvature scalar, with the "supermetric" $\mathcal{G}_{i j k l}$ given by

$$
\mathcal{G}_{i j k l} \equiv \frac{1}{2} g^{-1 / 2}\left(g_{i k} g_{j l}+g_{i l} g_{j k}-g_{i j} g_{k l}\right),
$$

while (; is the covariant derivative with respect to $g_{i j}$ )

$$
\mathcal{H}^{i} \equiv-2 \pi_{; j}^{i j}
$$

We note that, a priori, also $N$ and $N_{i}$ can be expanded in (5); while the background terms $N^{(0)}$ and $N_{i}^{(0)}$ are fixed functions, subject to the asymptotic conditions (2), the higher order terms represent true fluctuations in the lapse function 
and the shift vector: variations of $N$ and $N_{i}$ yield, at the classical level, the constraints:

$$
\begin{array}{r}
\mathcal{H}=\sum_{n \geq 1} \mathcal{H}^{(n)}=0 \\
\mathcal{H}^{i}=\sum_{n \geq 1} \mathcal{H}^{i(n)}=0,
\end{array}
$$

leaving in the expression of the classical energy only the $E_{A D M}^{(\circ)}$ term.

Since $\eta_{i j}, N^{(0)}$ and $N_{i}^{(0)}$ form together a solution $\eta_{\mu \nu}$ of matterless Einstein's equations, the linear term in the canonical Lagrangian density must be a total divergence. In our case, where $\eta_{\mu \nu}$ is static, this is a purely spatial divergence and, keeping the asymptotic flatness of background in mind, it must necessarily coincide with $E_{A D M}^{(1)}$. Thus, we have:

$$
E_{A D M}^{(1)}=\frac{1}{16 \pi G} \int_{\partial \Sigma} d S^{k}\left(h_{k j, j}-h_{j j, k}\right)=-\int_{\Sigma} d^{3} x\left(N^{(0)} \mathcal{H}^{(1)}+N_{i}^{(0)} \mathcal{H}^{i(1)}\right),
$$

that, together with the constraint (9) allows us to rewrite the energy (5) as

$$
E=E_{A D M}^{(0)}+\sum_{n \geq 2} \int_{\Sigma} d^{3} x\left(N^{(0)} \mathcal{H}^{(n)}+N_{i}^{(0)} \mathcal{H}^{i(n)}\right) .
$$

Note the survival of only the classical (0)-order terms in $N$ and $N_{i}$.

The quantization of the theory promotes the canonical pair $h_{i j}(x), \pi^{k l}(y)$ on $\Sigma$ to operators obeying the commutation rules:

$$
\left[h_{i j}(x), \pi^{k l}(y)\right]=i \hbar \delta_{i}^{(k} \delta_{j}^{l)} \delta^{(3)}(x-y),
$$

and acting on a Hilbert space of functionals $\Psi$ that are annihilated by the constraints (9). The evolution of the physical states $\Psi$ is governed by the "Schrödinger equation"

$$
i \hbar \partial_{t} \Psi=E\left[h_{i j}, \pi^{k l}\right] \Psi
$$

where the Hamiltonian operator is given by (11). Note that this is just the description of the quantum dynamics made by the asymptotic observer at infinity.

We point out that our definition of the Hilbert space is truly consistent within our restriction of phase space to two pairs of canonical operators, obtained by the gauge conditions (that respect (91)). This does not mean a loss of invariance (and of physical reality) at all: despite its look, the total energy (11) is nothing but a rearrangement of the invariant ADM-energy.

We should also be aware that the configuration space representation of the canonical operators

$$
\begin{aligned}
& h_{i j}(x) \quad h_{i j}(x) \times \\
& \pi^{i j}(x) \longrightarrow-i \hbar \frac{\delta}{\delta h_{i j}(x)}
\end{aligned}
$$


acting on state functionals

$$
\Psi \longrightarrow \Psi\left[h_{k l}\right](t)
$$

is not easily manageable beyond the 1-loop level, where connected ghost terms appear. Beside that, for $n \geq 3$ the expansion of the Hamiltonian operator (11) contains products of conjugate operators, thus posing an ordering problem. These problems are related to the bad ultraviolet divergences that would still yield a non-renormalizable behaviour, the possible solution of which emerges from the results of Section 6, which show that the structure of the vacuum is, with good probability, essentially discontinuous at the Planck scale $l_{p}$. Thus, in the rest of our analysis the QFT we shall work with will be cut-off at the Planck scale, having clearly in mind that our results will only be meaningful if consistent with this fundamental assumption (see Section 6).

As for the constraints (9), the problems are easier to solve. In fact we first notice that at the lowest order, the Hamiltonian operator retains only quadratic terms in the fields, on which we have to impose consistently first order constraints, that do not annihilate the quantum energy. The following terms in the expansions (9) can be enforced through a systematic correction of the state functional $\Psi$ that readapts non-physical degrees of freedom order by order, thus not affecting the dynamics based on the degrees of freedom (two for each space point) isolated at the lowest level. $\mathrm{O}$ Thus in spite of the problems typical of Quantum Gravity, the parallelism with the situation in QCD [2] is fully regained.

According to our fundamental assumption to cut the theory at the Planck scale, we shall perform a 1-loop calculation, with the Hamiltonian operator truncated at $n=2$. We simply adopt the representation (14) and (15), thus obtaining the Schrödinger equation

$$
i \hbar \partial_{t} \Psi\left[h_{i j}\right](t)=H\left[h_{i j},-i \hbar \frac{\delta}{\delta h_{k l}}\right] \Psi\left[h_{i j}\right](t),
$$

where

$$
H=M+\int_{\Sigma} d^{3} x N^{(0)} \mathcal{H}^{(2)}\left(h_{i j}(x),-i \hbar \frac{\delta}{\delta h_{i j}(x)}\right),
$$

with $\Psi\left[h_{i j}\right](t)$ annihilated by the first order constraints

$$
\begin{aligned}
\mathcal{H}^{(1)}\left(h_{i j}(x),-i \hbar \frac{\delta}{\delta h_{i j}(x)}\right) \Psi\left[h_{i j}\right](t) & =0 \\
\mathcal{H}^{i(1)}\left(h_{i j}(x),-i \hbar \frac{\delta}{\delta h_{i j}(x)}\right) \Psi\left[h_{i j}\right](t) & =0 .
\end{aligned}
$$

Setting, as usual,

$$
\Psi\left[h_{i j}\right](t)=e^{-i E t / \hbar} \Psi\left[h_{i j}\right],
$$

\footnotetext{
${ }^{2}$ See for example the procedure followed in Appendix B of ref [2].
} 
the problem can be reduced to the eigenvalue equation

$$
H\left[h_{i j},-i \hbar \frac{\delta}{\delta h_{i j}}\right] \Psi\left[h_{i j}\right]=E \Psi\left[h_{i j}\right] .
$$

We can now investigate the ground state of the theory. Instead of solving directly the eigenvalue equation, we look for the minimization of the expectation value of $H$ on a class of gaussian wave-functionals:

$$
E^{(2)} \equiv \int[\mathcal{D} h] \Psi^{*}\left[h_{i j}\right] H\left[h_{i j},-i \hbar \frac{\delta}{\delta h_{i j}}\right] \Psi\left[h_{i j}\right] .
$$

If the background is stable under the action of quantum fluctuations, at the 1-loop level this result coincides with the solution of (20); if, on the contrary, simple minimization leads to an imaginary part in $E^{(2)}$, then we have discovered an unstable configuration, whose physical meaning must be investigated. We demonstrate in the next section that the latter case occurs when $h_{i j}$ fluctuate around the "wormhole solution" $\eta_{i j}$ discovered by Schwarzschild in 1916 [3], whose line elements in polar coordinates are given by $(2 G M<r<+\infty)$

$$
d s^{2}=-\frac{r-2 G M}{r} d t^{2}+\frac{r}{r-2 G M} d r^{2}+r^{2}\left(d \theta^{2}+\sin ^{2} \theta d \phi^{2}\right)
$$

and depend on the single parameter $M$, the ADM-mass, such that

$$
E_{A D M}^{(0)}=M \text {. }
$$

\section{Quantum Fluctuations on a Schwarzschild Background}

We shall now address the problem to evaluate the expectation value on a gaussian trial functional of the Hamiltonian (17) where, according to our fundamental hypothesis (to be checked for consistency at the end of the calculation), we keep only the quadratic terms in the field quantum fluctuations $h_{i j}$. This truncation corresponds to the one-loop approximation. From a classical standpoint this amounts to a calculation of the energy carried by the quantized gravitational waves propagating on a fixed background, in the weak field approximation.

In our analysis we shall follow closely the steps of the ref. [2], where a similar calculation was carried out for a Yang-Mills theory. We begin by constructing the Hilbert space of the states of the gravitational field, introducing the following scalar product:

$$
\left\langle\left\langle\Psi \mid \Psi^{\prime}\right\rangle\right\rangle \equiv \int(\mathcal{D} h) \triangle_{F P} \Psi^{*}\left[h_{i j}\right] \Psi^{\prime}\left[h_{i j}\right]
$$

where $(\mathcal{D} h)$ denotes the measure of the functional space and $\triangle_{F P}$ represents the Fadeev-Popov determinant, depending on the gauge adopted, necessary to 
recuperate the gauge-invariance, i.e. the general covariance of QG. The Hilbert space will thus be the space of the state-vectors $\Psi\left[h_{i j}\right](t)$ that with the metric (24) are normalizable. We note that for an infinitesimal coordinate transformation the quantum field $h_{i j}$ gets transformed as:

$$
h_{i j}(x) \longrightarrow h_{i j}(x)-\xi_{i \mid j}(x)-\xi_{j \mid i}(x),
$$

just like a weak classical field. And in our approximation, being the gaugeconditions (9) linear in the field $h_{i j}$, the determinant $\triangle_{F P}$ does not depend on $h_{i j}$ and can be therefore neglected. For a generic operator $\hat{O}\left[h_{i j}, \pi^{i l}\right]$, the expectation value on a state $\Psi\left[h_{i j}\right](t)$ can be defined as:

$$
\langle\langle\hat{O}\rangle\rangle_{\Psi}(t) \equiv \frac{\langle\langle\Psi \mid \hat{O} \Psi\rangle\rangle}{\langle\langle\Psi \mid \Psi\rangle\rangle} .
$$

Let us consider now a hypersurface $\Sigma$ at a fixed time $t$. We wish to compute the expectation value of the (truncated) Hamiltonian on the gaussian trial functional:

$$
\Psi_{G}\left[h_{i j}\right]=\exp -\frac{1}{4} \int_{x, y} h_{i j}(\vec{x}) \Gamma^{i j k l}(\vec{x}, \vec{y}) h_{k l}(\vec{y})
$$

where $\int_{x} \equiv \int_{\Sigma} d^{3} \vec{x} \eta^{1 / 2}(\vec{x})$ and $\eta^{\frac{1}{2}}(\vec{x})=\sqrt{\frac{r}{r-2 m}}(m=M G$ is the one half Schwarzschild radius). In order to get a normalizable $\Psi_{G}\left[h_{i j}\right]$ we require that $\Gamma^{i j k l}(\vec{x}, \vec{y})$ be real and positive, symmetric under the exchanges $i \leftrightarrow j, k \leftrightarrow l, i j, \vec{x} \leftrightarrow k l, \vec{y}$.

The second order Hamiltonian density is given by

$$
\mathcal{H}^{(2)}(\vec{x})=T^{(2)}(\vec{x})+V^{(2)}(\vec{x})
$$

with

$$
\begin{gathered}
T^{(2)}=-16 \pi G \eta^{-1 / 2}\left(\eta^{i k} \eta^{j l}-\frac{1}{2} \eta^{i j} \eta^{k l}\right) \frac{\delta^{2}}{\delta h_{i j}(\vec{x}) \delta h_{k l}(\vec{x})} \\
V^{(2)}=-\frac{1}{16 \pi G} \eta^{1 / 2}\left(R^{(2)}+\frac{1}{2} h_{k}^{k} R^{(1)}\right) .
\end{gathered}
$$

We observe that for a Gaussian wave-functional the expectation value of two fields is given by:

$$
\left\langle\left\langle h_{i j}(\vec{x}) h_{k l}(\vec{y})\right\rangle\right\rangle_{\Psi}=G_{i j k l}(\vec{x}, \vec{y})
$$

where $G_{i j k l}(\vec{x}, \vec{y})$ satisfies the relationship:

$$
\int_{z} \Gamma^{i j m n}(\vec{x}, \vec{z}) G_{m n k l}(\vec{z}, \vec{y})=\eta^{-1 / 2}(\vec{y}) \delta_{(k}^{i} \delta_{l)}^{j} \delta^{(3)}(\vec{x}-\vec{y}) .
$$

In this way one gets for the expectation value of the Hamiltonian 3

$$
\langle\langle H\rangle\rangle_{\Psi}=M+\hbar \int_{x} N(\vec{x})\left[\frac{\alpha}{4}\left(\Gamma^{i j}{ }_{i j}(\vec{x}, \vec{x})-\frac{1}{2} \Gamma_{i j}^{i j}(\vec{x}, \vec{x})\right)+\frac{1}{\alpha} \hat{O}^{i j k l}(\vec{x}) G_{k l i j}(\vec{x}, \vec{x})\right],
$$

\footnotetext{
${ }^{3}$ In order to clearly separate the classical from the quantum (one-loop) contributions for the rest of this Section we shall keep the Planck constant $\hbar$, instead of putting it equal to one, as done in the natural unit system.
} 
where

$$
\alpha \equiv 16 \pi G \hbar=16 \pi l_{P}^{2},
$$

and the differential operator $\hat{O}^{i j k l}(\vec{x})$ is defined by

$$
\int_{x} N V^{(2)}=\frac{1}{\alpha} \int_{x} N(\vec{x}) h_{i j}(\vec{x}) \hat{O}^{i j k l}(\vec{x}) h_{k l}(\vec{x}),
$$

and represents the "potential" contribution to the quadratic Hamiltonian (17). In order to guarantee the general covariance of our calculation, it is necessary to impose on the physical state the quantum constraints, which in our approximation are

$$
\begin{gathered}
\mathcal{H}^{(1)} \Psi=\left(h^{i j} R_{i j}^{(0)}-h^{i j}{ }_{\mid i j}-h_{i \mid j}^{i}{ }^{\mid j}\right) \Psi=0 \\
\mathcal{H}^{i(1)} \Psi=\pi_{\mid j}^{i j} \Psi=0
\end{gathered}
$$

which are obeyed provided,

$$
\nabla_{j}(\vec{x}) \Gamma^{i j k l}(\vec{x}, \vec{y})=0
$$

where $\nabla_{j}(\vec{x})$ denotes the covariant derivative with respect to the background field; and, fixing the lapse function and the shift vector as

$$
N=N^{(0)}=\sqrt{1-\frac{2 m}{r}}, N^{i}=N^{i(0)}=0,
$$

we have

$$
\nabla_{i}\left(\frac{h_{j}^{i}}{N}\right)=0, h_{k}^{k}=0 .
$$

By consistency with the trace condition $h_{k}^{k}=0$ we must also impose $\pi_{k}^{k} \Psi=0$, which yields the further constraint:

$$
\Gamma_{i}^{i k l}(\vec{x}, \vec{y})=\Gamma_{k}^{i j k}(\vec{x}, \vec{y})=0 .
$$

The elements of the Hilbert space of the physical modes of the gravitational field $h_{i j}$ are thus the symmetric tensors of rank $2 \phi_{i j}$, defined in $\Sigma$, obeying the boundary conditions (2) and the gauge conditions:

$$
\nabla_{i}\left(\frac{\Phi_{j}^{i}}{N}\right)=0, \Phi_{k}^{k}=0
$$

normalizable with respect to the scalar product:

$$
\left\langle\Phi \mid \Phi^{\prime}\right\rangle \equiv \int_{x} N^{-1}(\vec{x}) \Phi^{* i j}(\vec{x}) \Phi_{i j}^{\prime}(\vec{x}) .
$$


In this way we may construct in our Hilbert space a complete orthogonal system, by making use of the spectral decomposition of the operator $\hat{O}^{i j k l}(\vec{x})$ or, better,. of the operator $\hat{Q}^{i j k l}$ defined as:

$$
\int_{x} N V^{(2)}=\frac{1}{\alpha} \int_{x} N(\vec{x}) h_{i j}(\vec{x}) \hat{O}^{i j k l}(\vec{x}) h_{k l}(\vec{x})=\int_{x} N^{-1}(\vec{x}) h_{i j}(\vec{x}) \hat{Q}^{i j k l}(\vec{x}) h_{k l}(\vec{x}),
$$

where in order to go from $\hat{O}$ to $\hat{Q}$ a total divergence has been added to the integrand, without changing the "potential" contribution to the Hamiltonian. Thus the operator $\hat{Q}^{i j k l}$ becomes in our Hilbert space a self-adjoint hermitian operator, whose eigenfunctions ( $\rho$ denotes a complete set of indices),

$$
\hat{Q}_{i j}{ }^{k l} \Phi_{k l}^{(\rho)}=\lambda(\rho) \Phi_{i j}^{(\rho)},
$$

build the sought complete orthonormal basis. In this basis the "propagator" $G_{i j k l}(\vec{x}, \vec{y})$ has the simple form;

$$
G_{i j k l}(\vec{x}, \vec{y})=\sum_{\rho} \frac{1}{2 f(\rho)} \Phi_{i j}^{(\rho) *}(\vec{x}) \Phi_{k l}^{(\rho)}(\vec{y}),
$$

where $f(\rho)$ denotes a set of variational parameters to be determined by the minimization of the expectation value (33).

From (32) and (46) we obtain,

$$
\Gamma^{i j k l}(\vec{x}, \vec{y})=\sum_{\rho} 2 f(\rho) N^{-1}(\vec{x}) N^{-1}(\vec{y}) \phi_{(\rho)}^{* i j}(\vec{x}) \phi_{(\rho)}^{k l}(\vec{y}) .
$$

We may now easily compute the expectation value (33), and obtain:

$$
\langle\langle H\rangle\rangle_{\Psi}=M+\frac{\hbar}{2} \sum_{\rho}\left(\alpha f(\rho)+\frac{\lambda(\rho)}{\alpha f(\rho)}\right) .
$$

and minimizing with respect to the variational function $f(\rho)$, i.e. imposing

$$
\frac{\delta}{\delta f(\rho)}\langle\langle H\rangle\rangle_{\Psi}=0
$$

we readily get

$$
f(\rho)=\alpha^{-1} \sqrt{\lambda(\rho)}
$$

which inserted in (48) finally yields:

$$
E=M+\hbar \sum_{\rho} \sqrt{\lambda(\rho)}
$$

All the above makes sense if and only if

$$
\lambda(\rho)>0
$$


i.e. the eigenvalue of the "potential" operator $\hat{Q}$ are positive definite. If, instead, for some $\rho \lambda(\rho) \leq 0$ the one-loop approximation, yielding imaginary contributions to the energy of the ground state, breaks down, showing that the Perturbative Ground State (PGS, $M \rightarrow 0$ ) is essentially unstable.

This is precisely the situation found in the study of $S U(n)$ Yang-Mills theories [2] where, going beyond the one-loop approximation, one could easily check that the modes belonging to the sector where $\lambda(\rho) \leq 0$ did not contribute to the energy of the state terms of $O(\hbar)$, but rather of $O(1)$, just like the classical term $M$. This "promotion" of a quantum $O(\hbar)$ contribution to a classical $O(1)$ one, can be understood when we realize that the amplitude $f(\rho)^{-\frac{1}{2}}$ of the modes with $\lambda(\rho) \leq 0$ (the "unstable modes")[see Eqs.(31) and (46)] is only prevented from becoming infinite by the neglected positive terms of $O\left(\hbar^{2}\right)$. In this way $\alpha f(\rho)$ becomes $O\left(\frac{1}{\hbar}\right)$ and the negative contribution from the "unstable modes" is just classical, i.e. $O(1)$.

In the calculation of Ref.[2] one could explicitly prove that this "promoted" quantum contribution completely screens the classical positive term (such as, in our case $M$ ), thus realizing a "vacuum" state whose energy density is way that of below the PGS, which as a result becomes unstable at all space-time scales. In the case of QG the problem of going beyond the one-loop approximation is formidable, utterly beyond our present means of analysis, however, as shall be discussed below, to figure out the contributions to the energy of the trial states of possible "unstable modes" with $\lambda(\rho) \leq 0$ appears reasonably doable.

In order to precede further we must first compute the operator $\hat{Q}^{i j k l}(\vec{x})$ and then diagonalize it, which we shall do next. Defining $\bar{h}^{i j} \equiv g^{i j}-\eta^{i j}$, we have:

$$
\bar{h}^{i j}=-h^{i j}+h_{l}^{i} h^{l j}-h_{l}^{i} h_{m}^{l} h^{m j}+\ldots
$$

and for the perturbative calculations it is useful to introduce:

$$
S_{m n}^{a}=\Gamma_{m n}^{a}-\Gamma_{m n}^{a(s)}
$$

which turns out to be tensor.

Observing that (";" denotes the full covariant derivative, while "" is the covariant derivative with respect to the Schwarzschild background)

$$
h_{j a ; i}=h_{j k \mid i}-S_{i m}^{l} h_{l j}-S_{i j}^{l} h_{l k}
$$

we may write the Riemann and the Ricci tensors as:

$$
\left\{\begin{array}{l}
R_{b c d}^{a}=R_{b c d}^{a(s)}+S_{b d \mid c}^{a}-S_{b c \mid d}^{a}+S_{m c}^{a} S_{b d}^{m}-S_{d m}^{a} S_{b c}^{m} \\
R_{b c}=R_{b c}^{(s)}+S_{b c \mid a}^{a}-S_{a b \mid c}^{a}+S_{a m}^{a} S_{b c}^{m}-S_{l b}^{a} S_{a c}^{l}
\end{array}\right.
$$

\footnotetext{
${ }^{4}$ Like it happens, according to $(51)$, to the modes belonging to the "stable sector", for which $\lambda(\rho)>0$.
} 
furthermore:

$$
S_{j k}^{i}=\frac{1}{2} g^{i k}\left(h_{j r \mid k}+h_{r k \mid j}-h_{j k \mid r}\right) .
$$

We may now compute the curvature scalar $R=g^{b c} R_{b c}$, which for convenience we decompose as the sum of three terms:

$$
A \equiv g^{b c} R_{b c}^{(s)} ; \quad B \equiv g^{b c} S_{b c \mid l}^{l}-g^{b c} S_{l b \mid c}^{l} ; \quad C \equiv g^{b c}\left(S_{l m}^{l} S_{b c}^{m}-S_{m b}^{l} S_{l c}^{m}\right) .
$$

In this way to first order in $h_{i j}$ we have:

$$
A^{(1)}=\frac{m}{r^{3}}\left(-h+3 h_{1}^{1}\right)
$$

while to second order we obtain:

$$
A^{(2)}=\frac{m}{r^{3}}\left(h_{i}^{a} h_{a}^{i}-3 h_{i}^{1} h_{1}^{i}\right) .
$$

As for $B$ we have

$$
B^{(1)}=\eta^{l m} S_{l m \mid a}^{a(1)}-\eta^{l m} S_{l a \mid m}^{a(1)}=h_{\mid b a}^{a b}-h_{\mid a}^{a}
$$

and

$$
\begin{gathered}
B^{(2)}=\left\{-\left[h^{a r} h_{r s}^{\mid s}\right]_{\mid a}+\frac{1}{2} h_{\mid a}^{a r} h_{\mid r}-h^{l m} h_{m \mid a l}^{a}\right\}+\left\{h^{l m} h_{\mid m}-2 h A^{(1)}\right\} \\
-3 A^{(2)}+h^{l m} h_{l m a}{ }_{l a}+\frac{1}{2} h_{\mid r}^{l m} h_{l m}{ }^{\mid r}
\end{gathered}
$$

respectively. While for the third term one has:

$$
C^{(2)}=\frac{1}{2} h_{\mid b} h_{\mid a}^{b a}-\frac{1}{4} h_{\mid b} h^{\mid b}-\frac{1}{4}\left(2 h^{a l \mid b} h_{a b \mid l}-h^{a m \mid b} h_{a m \mid b}\right) .
$$

By summing the different terms we obtain the following expansion of the scalar curvature:

$$
\begin{gathered}
R^{(0)}=0 \\
R^{(1)}=\left\{h_{\mid b a}^{a b}\right\}+\left\{-\frac{m}{r^{3}} h-h_{\mid a}^{a}\right\}+\left\{3 \frac{m}{r^{3}} h_{1}^{1}\right\} \\
R^{(2)}=\left\{-\left[h^{a r} h_{r s}^{\mid s}\right]_{\mid a}+h_{\mid a}^{a r} h_{\mid r}-h^{l m} h_{m \mid a l}^{a}\right\} \\
+\left\{h^{l m} h_{\mid l m}+2(h)^{2} \frac{m}{r^{3}}-6 \frac{m}{r^{3}} h h_{1}^{1}-\frac{1}{4} h_{\mid b} h^{\mid b}\right\} \\
+\left\{-\frac{2 m}{r^{3}}\left(h_{i}^{a} h_{a}^{i}-3 h_{i}^{1} h_{1}^{i}\right)+h^{l m} h_{l m a} \mid a\right. \\
\left.+\frac{3}{4} h_{\mid r}^{l m} h_{l m}{ }_{\mid r}-\frac{1}{2} h_{\mid b}^{a l} h_{a \mid l}^{b}\right\} .
\end{gathered}
$$


But we are not done yet, we must expand the square root of the determinant of the metric $\sqrt{\|g\|}$. Due to $R^{(0)}=0$ we may stop at the first order, obtaining:

$$
\sqrt{\frac{\|\boldsymbol{g}\|}{\|\boldsymbol{\eta}\|}}=1+\frac{1}{2} h_{i}^{i}+O\left(h^{2} .\right)
$$

In this way for $V^{(2)}$ we get the following expansion:

$$
\begin{aligned}
V^{(2)} & =-\frac{1}{16 \pi G} \sqrt{\|\boldsymbol{\eta}\|}\left[\frac{1}{2} h_{\mid b a}^{a b} \cdot h-\frac{1}{2} \frac{m}{r^{3}}(h)^{2}-\frac{1}{2} h_{\mid a}{ }^{a}\right. \\
& +3 \frac{m}{r^{3}} h_{1}^{1} h-\left(h^{a r} h_{r s}{ }_{\mid s}\right)_{\mid a}+h^{a r}{ }_{\mid a} h_{\mid r}-h^{l m} h_{m \mid a l}^{a}+h^{l m} h_{\mid l m} \\
& +2(h)^{2} \frac{m}{r^{3}}-6 \frac{m}{r^{3}} h h_{1}^{1}-\frac{1}{4} h_{\mid b} h^{\mid b}-\frac{2 m}{r^{3}}\left(h_{i}^{a} h_{a}^{i}-3 h_{i}^{1} h_{1}^{i}\right) \\
& \left.+h^{l m} h_{l m}{ }_{\mid a}+\frac{3}{4} h_{\mid r}^{l m} h_{l m}{ }_{\mid r}-\frac{1}{2} h_{\mid b}^{a l} h_{a \mid l}^{b}\right] .
\end{aligned}
$$

We are now ready to compute the operator $\hat{Q}^{i j k l}$. Let us fix the gauge as: $h=0$ and $\left(\frac{h^{i j}}{N}\right)_{\mid j}=0$, the latter condition by use of the former giving

$$
h_{i \mid j}^{j}=\frac{m}{r^{2}} h_{1 i} .
$$

We also note that given a vector $T^{m}$, one has

$$
\int_{x} N\left[T^{m}\right]_{\mid m}=-\int_{x} N_{, m} T^{m}=-\int_{x} T^{1} \frac{1}{\sqrt{1-\frac{2 m}{r}}} \frac{m}{r^{2}}=\int_{x} T^{1} N \Gamma_{11}^{1} .
$$

Thus we obtain for the integral of the second order potential,

$$
\begin{aligned}
\int_{x} N V^{(2)} & =\int_{x} N \frac{1}{4} h_{b}^{a}\left(-\nabla^{2} h_{a}^{b}+3 h_{a \mid 1}^{b} \frac{m}{r^{2}}-h_{a \mid} \mid b \frac{m}{r^{2}}-h_{1 \mid a}^{b} \frac{m}{r^{2}}\right. \\
+h_{c}^{b} R_{a}^{c} & \left.+h_{a}^{c} R_{c}^{b}-\frac{8 m^{2}}{r^{3}(r-2 m)} \delta_{(a}^{1} h_{1}^{b)}\right) .
\end{aligned}
$$

Recalling Eq.(44), Eq.(45) becomes:

$$
\begin{gathered}
N^{2}\left[-\nabla^{2} \phi_{a}^{b}+3 \phi_{a \mid 1}^{b} \frac{m}{r^{2}}-\phi_{a 1}{ }^{\mid b} \frac{m}{r^{2}}-\phi_{1 \mid a}^{b} \frac{m}{r^{2}}+\phi_{c}^{b} R_{a}^{c}+\phi_{a}^{c} R_{c}^{b}\right. \\
\left.-\frac{8 m^{2}}{r^{3}(r-2 m)} \delta_{(a}^{1} h_{1}^{b)}\right]+v_{\mid a}^{b}+v_{a}^{\mid b}+\eta_{a}^{b} \tau=4 \lambda \phi_{a}^{b},
\end{gathered}
$$

where we have added the terms in $v^{i}$ e $\tau$ in order to remain inside the Hilbert space of the tensors $\phi^{i j}$, which obey the conditions [see Eq.(42)]

$$
\begin{gathered}
\phi_{i}^{i}=0 \\
\phi_{i \mid j}^{j}=\frac{m}{r^{2}} \phi_{1 i} .
\end{gathered}
$$


By taking the trace of eq.(71) with $\eta_{b}^{a}$ we get immediately that

$$
\tau=-\frac{2}{3} v_{\mid b}^{b}-N^{2}\left(R_{1}^{1} \phi_{1}^{1}-\frac{10 m^{2}}{3 r^{3}} \frac{\phi_{1}^{1}}{r-2 m}\right) .
$$

By consistency, we must require that the terms in $v_{i}$ do not contribute to the potential energy, this obviously implies that

$$
\int_{x}\left[N^{-1} \phi_{(\rho)}^{i j} v_{i}^{(\rho)}\right]_{\mid j}=0,
$$

which requires that for $r \rightarrow \infty$ the following conditions be satisfied:

$$
\begin{aligned}
v_{1} & \rightarrow O\left(r^{-1}\right) \\
v_{2}, v_{3} & \rightarrow O(1),
\end{aligned}
$$

and

$$
\lim _{r \rightarrow+\infty} r^{2} \int d \Omega \phi_{1}^{i} v_{i}=\lim _{r \rightarrow 2 m} r^{2} \int d \Omega \phi_{1}^{i} v_{i}=0
$$

\section{The eigenvalues and eigenmodes of the sec- ond order potential}

Due to the spherical symmetry of the Schwarzschild background a particularly suitable method to obtain the solutions of the eigenvalue problem posed by Eq.(71) is the one devised by T. Regge and J.A. Wheeler [州, for the study of the small (classical) fluctuations around the Schwarzschild solution. By making use of this method the eigenfunction are separated in two classes (see appendix B), the "even" solutions with parity $(-1)^{l}$, equal to the parity of spherical harmonics $Y_{l m}(\theta, \phi)$, and the "odd" solutions with opposite parity.For the "even" solutions, if we set

$$
\left\{\begin{aligned}
\phi_{1}^{1} & =H(r) Y_{l m}(\theta, \varphi) \\
\phi_{2}^{2} & =\left(G_{1}(r)+G_{2}(r) \partial_{\theta}^{2}\right) Y_{l m}(\theta, \varphi) \\
\phi_{3}^{3} & =\left(G_{1}(r)+\frac{G_{2}(r)}{\sin ^{2} \theta} \partial_{\varphi}^{2}+G_{2}(r) \cot \theta \partial_{\theta}\right) Y_{l m}(\theta, \varphi) \\
\phi_{1}^{2} & =K(r) \partial_{\theta} Y_{l m}(\theta, \varphi) \\
\phi_{1}^{3} & =\frac{K(r)}{\sin ^{2} \theta} \partial_{\varphi} Y_{l m}(\theta, \varphi) \\
\phi_{2}^{3} & =\frac{G_{2}(r)}{\sin ^{2} \theta}\left(\partial_{\theta}-\cot \theta\right) \partial_{\varphi} Y_{l m}(\theta, \varphi),
\end{aligned}\right.
$$

the eigenvalue equations will turn out to be, as we shall see in a moment, completely factorized. As for the vector $v_{i}(71)$, factorization is achieved if we set,

$$
\left\{\begin{array}{l}
v_{1}=U(r) Y_{l m}(\theta, \varphi) \\
v_{2}=V(r) \partial_{\theta} Y_{l m}(\theta, \varphi) \\
v_{3}=V(r) \partial_{\varphi} Y_{l m}(\theta, \varphi)
\end{array}\right.
$$


Substituting (77) and (78) in (71) (see appendix C) we obtain a system of differential equations for the radial functions only:

$$
\left\{\begin{aligned}
& N^{2}\left\{\left[\frac{r-2 m}{r} \partial_{r}^{2}+2 \frac{r-2 m}{r^{2}} \partial_{r}-\frac{2}{3} \frac{6 r^{2}-27 m r+23 m^{2}}{r^{3}(r-2 m)}\right] H(r)\right. \\
&+\left.4 \frac{r-2 m}{r^{2}} l(l+1) K(r)+2 \frac{r-2 m}{r^{3}}\left(2 G_{1}(r)-l(l+1) G_{2}(r)\right)\right\} \\
&=-H(r) 4 \lambda+\frac{4}{3}\left[\frac{r-2 m}{r} \partial_{r} U(r)-\frac{r-3 m}{r^{2}} U(r)+\frac{V(r)}{2} \frac{l(l+1)}{r^{2}}\right] \\
&+N^{2} \frac{l(l+1)}{r^{2}} H(r) \\
& N^{2}\left\{\left[\frac{r-2 m}{r} \partial_{r}^{2}+2 \frac{r-3 m}{r^{2}} \partial_{r}+\frac{2}{r^{2}}\right] G_{2}(r)+2 \frac{2 r-3 m}{r^{2}} K(r)\right\} \\
&=-4 \lambda G_{2}(r)+\frac{2 V(r)}{r^{2}}+N^{2} \frac{l(l+1)}{r^{2}} G_{2}(r) \\
& N^{2}\left\{\left[\frac{r-2 m}{r} \partial_{r}^{2}+2 \frac{r-3 m}{r^{2}} \partial_{r}-\frac{2}{r^{2}}\right] G_{1}(r)+\frac{2}{r^{2}} G_{2}(r) l(l+1)\right. \\
&\left.+\quad \frac{2}{3} \frac{3 r^{2}-12 m r+7 m^{2}}{r^{3}(r-2 m)} H(r)\right\} \\
&=-4 \lambda G_{1}(r)-\frac{2}{3}\left[\frac{r-2 m}{r} \partial_{r} U(r)-\frac{r-3 m}{r^{2}} U(r)-\frac{V(r)}{r^{2}} l(l+1)\right] \\
&+ N^{2} \frac{l(l+1)}{r^{2}} G_{1}(r) \\
& N^{2}\left\{\left[\frac{r-2 m}{r} \partial_{r}^{2}+\frac{4 r-7 m}{r^{2}} \partial_{r}-\frac{2 r^{2}-m r \cdot 10+10 m^{2}}{r^{3}(r-2 m)}\right] K(r)\right. \\
&+\left.\frac{2 r-3 m}{r^{3}(r-2 m)} H(r)-\frac{2}{r^{3}}\left[G_{1}(r)+(1-l(l+1)) G_{2}(r)\right]\right\} \\
&=-4 \lambda K(r)+\frac{1}{r^{2}}\left(U(r)+\partial_{r} V(r)-\frac{2}{r} V(r)\right)+N^{2} \frac{l(l+1)}{r^{2}} K(r) .
\end{aligned}\right.
$$

And by substituting the same expansions in the constraint Eq.(42), we obtain,

$$
\begin{gathered}
2 G_{1}(r)-G_{2}(r) l(l+1)+H(r)=0 \\
\partial_{r} H(r)+\frac{3 H(r)}{r}-\frac{m}{r(r-2 m)} H(r)-K(r) l(l+1)=0 \\
\partial_{r} K(r) r(r-2 m)+K(r)(4 r-8 m) \\
+G_{1}(r)+G_{2}(r)[1-l(l+1)]=0 .
\end{gathered}
$$

The boundary conditions (75) for $r \rightarrow \infty$ become:

$$
\left\{\begin{array}{l}
U(r) \rightarrow O\left(\frac{1}{r}\right) \\
V(r) \rightarrow O(1)
\end{array}\right.
$$

while (76) imply:

$$
\begin{aligned}
& \left.\left\{r^{2}[H(r) \cdot U(r)+l(l+1) K(r) V(r)]\right\}\right|_{r=2 m} \\
& \quad=\left.\left\{r^{2}[H(r) \cdot U(r)+l(l+1) K(r) V(r)]\right\}\right|_{r=+\infty}=0 .
\end{aligned}
$$


Proceeding in a completely analogous way, for the "odd" solutions one gets:

$$
\left\{\begin{array}{l}
\phi_{1}^{1}=0 \\
\phi_{2}^{2}=F_{2}(r) \frac{1}{\sin \theta}\left[\partial_{\theta}-\cot \theta\right] \partial_{\varphi} Y_{l m}(\theta, \varphi) \\
\phi_{3}^{3}=-\phi_{2}^{2} \\
\phi_{1}^{2}=-\frac{F_{1}(r)}{\sin \theta} \partial_{\varphi} Y_{l m}(\theta, \varphi) \\
\phi_{1}^{3}=\frac{F_{1}(r)}{\sin \theta} \partial_{\theta} Y_{l m}(\theta, \varphi) \\
\phi_{2}^{3}=\frac{1}{2} \frac{F_{2}(r)}{\sin \theta}\left[\frac{1}{\sin ^{2} \theta} \partial_{\varphi}^{2}+\cot \theta-\partial_{\theta}^{2}\right] Y_{l m}(\theta, \varphi)
\end{array}\right.
$$

and

$$
\begin{aligned}
& v_{1}=0 \\
& v_{2}=D(r)\left(-\frac{1}{\sin \theta}\right) \partial_{\varphi} Y_{l m}(\theta, \varphi) \\
& v_{3}=D(r) \sin \theta \partial_{\theta} Y_{l m}(\theta, \varphi) .
\end{aligned}
$$

And substituting these last expressions in the eigenvalue equations, we get the following relationships:

$$
\begin{gathered}
N^{2}\left[-\frac{r-2 m}{r} \partial_{r}^{2}-\frac{6 r-11 m}{r^{2}} \partial_{r}+\frac{l(l+1)}{r^{2}}+\frac{2}{r^{3}}(-3(r-2 m)\right. \\
\left.\left.-\frac{r-m}{r-2 m} m\right)\right] F_{1}(r)=4 \lambda F_{1}(r)+\frac{1}{r^{2}}\left(\partial_{r}-\frac{2}{r}\right) D(r)
\end{gathered}
$$

and

$$
\begin{aligned}
& N^{2}\left[\left(-\frac{r-2 m}{r} \partial_{r}^{2}-2 \frac{r-3 m}{r^{2}} \partial_{r}+\frac{l(l+1)}{r^{2}}-\frac{2}{r^{2}}\right) F_{2}(r)\right. \\
& \left.\quad+2 \frac{2 r-3 m}{r^{2}} F_{1}(r)\right]=4 \lambda F_{2}(r)-\frac{1}{r^{2}} D(r)
\end{aligned}
$$

Noting that the fluctuations' tensor automatically satisfies the traceless condition, we find that the gauge conditions yield

$$
(r-2 m)\left(r \partial_{r}+4\right) F_{1}(r)-\left(1-\frac{l(l+1)}{2}\right) F_{2}(r)=0
$$

as the only constraint. 
The conditions (75) reduce to $D(r) \rightarrow O(1)$, while (76) yield

$$
\left.\left[l(l+1) r^{2} F_{1}(r) D(r)\right]\right|_{r=2 m}=\left.\left[l(l+1) r^{2} F_{1}(r) D(r)\right]\right|_{r=+\infty}=0 .
$$

An important observation is that for S-waves $(l=0)$ the gauge conditions are sufficient to determine the form of the solution. In the other cases we shall solve the eigenvalue problem in the WKB approximation.

Setting now $l=0$ in our equation we realize at once that the "odd" part vanishes identically, while the first of the $(79)$ has a particularly simple form, depending on $H(r)$ and $G(r)$ only, the latter obeying through (80) $H(r)=-2 G_{1}(r)$. The fluctuations' tensor acquires thus the following simple form:

$$
\phi_{b}^{a}(r, \theta, \varphi)=\frac{1}{\sqrt{4 \pi}}\left(3 \delta_{1}^{a} \delta_{b}^{1}-\delta_{b}^{a}\right) \frac{1}{2} H(r),
$$

where $H(r)$ is determined via eq.(81)

$$
\partial_{r} H(r)+H(r)\left[\frac{3}{r}+\frac{1}{2}\left(\frac{1}{r}-\frac{1}{r-2 m}\right)\right]=0,
$$

whose solution is

$$
H(r)=A \sqrt{1-\frac{2 m}{r}} \frac{1}{r^{3}},
$$

where the constant $A$ is determined by the normalization condition.

$$
1=\langle\phi \mid \phi\rangle=\int_{\Sigma} d^{3} \vec{x}\|\boldsymbol{\eta}\|^{1 / 2} \frac{1}{N} \frac{1}{4 \pi} \frac{3}{2} H^{2}(r)=\frac{A^{2}}{16 m^{3}}
$$

which yields $A=4 m^{3 / 2}$, so that

$$
H(r)=4 m^{3 / 2} \sqrt{1-\frac{2 m}{r}} \frac{1}{r^{3}} .
$$

In Appendix D, we develop the calculation for the eigenfunctions' normalization in general.

It remains to be verified whether there exists an eigenvalue corresponding to (94), in other words, we are looking for a value $\lambda$ for which (94) is a solution of the eigenvalue equations satisfying the boundary conditions (75) and (76). On substituting (94) in the first of (79), and observing that

$$
\begin{gathered}
\partial_{r} H(r)=-\frac{3 r-7 m}{r(r-2 m)} H(r) \\
\partial_{r}^{2} H(r)=\frac{12 r^{2}-56 m r+63 m^{2}}{r^{2}(r-2 m)^{2}} H(r)
\end{gathered}
$$


and

$$
G_{1}(r)=-\frac{1}{2} H(r)
$$

we find:

$$
\begin{aligned}
& \frac{4}{3}\left(\frac{r-2 m}{r} \frac{d}{d r}-\frac{r-3 m}{r^{2}}\right) U(r) \\
& \quad=\left(4 \lambda-\frac{m(12 r-35 m)}{3 r^{4}}\right) 4 m^{3 / 2} \sqrt{1-\frac{2 m}{r}} \frac{1}{r^{3}}
\end{aligned}
$$

whose solution is:

$$
U(r)=\sqrt{\frac{r}{r-2 m}}\left[r C+4 m^{3 / 2}\left(-\frac{\lambda}{r^{2}}+\frac{m}{2 r^{5}}-\frac{5}{4} \frac{m^{2}}{r^{6}}\right)\right],
$$

$C$ being an integration constant. Due to (75) $C$ must vanish, while (76) finally fixes the eigenvalue $\lambda$. Indeed from

$$
\lim _{r \rightarrow 2 m} r^{2} H(r) U(r)=0
$$

one gets for the eigenvalue $\lambda$ :

$$
\lambda=-\frac{1}{64 m^{2}}=-\frac{1}{64(G M)^{2}}
$$

This finding of a negative eigenvalue $\lambda$, i.e. of an "unstable mode" around a "wormhole", in view of the discussion in section 3, is a most significant result of our analysis that strengthens the analogy of the present calculation with the QCD one 2]. We must, however, point out that (97) is not at all unexpected, since in ref. [5] a similar finding has been reported in a somewhat different context.

As for the remaining stable modes we shall solve the eigenvalue problem in an approximate way, by the WKB method. We shall thus obtain a good description in the semiclassical region. Let us then write our solution in the form

$$
\left(\begin{array}{c}
H(r) \\
K(r) \\
G_{1}(r) \\
G_{2}(r) \\
U(r) \\
V(r)
\end{array}\right)=\left(\begin{array}{c}
h(r) \\
k(r) \\
g_{1}(r) \\
g_{2}(r) \\
u(r) \\
v(r)
\end{array}\right) e^{\frac{i}{\hbar} S(r)}
$$




$$
\simeq\left(\begin{array}{c}
h_{0}(r)+\left(\frac{\hbar}{i}\right) h_{1}(r)+\left(\frac{\hbar}{i}\right)^{2} h_{2}(r)+\cdots \\
k_{0}(r)+\left(\frac{\hbar}{i}\right) k_{1}(r)+\left(\frac{\hbar}{i}\right)^{2} k_{2}(r)+\cdots \\
g_{10}(r)+\left(\frac{\hbar}{i}\right) g_{11}(r)+\left(\frac{\hbar}{i}\right)^{2} g_{12}(r)+\cdots \\
g_{20}(r)+\left(\frac{\hbar}{i}\right) g_{21}(r)+\left(\frac{\hbar}{i}\right)^{2} g_{22}(r)+\cdots \\
u_{0}(r)+\left(\frac{\hbar}{i}\right) u_{1}(r)+\left(\frac{\hbar}{i}\right)^{2} u_{2}(r)+\cdots \\
v_{0}(r)+\left(\frac{\hbar}{i}\right) v_{1}(r)+\left(\frac{\hbar}{i}\right)^{2} v_{2}(r)+\cdots \\
\cdot e^{\frac{i}{\hbar}\left(s_{0}(r)+\frac{\hbar}{i} s_{1}(r)+\left(\frac{\hbar}{i}\right)^{2} s_{2}(r)+\cdots\right)}
\end{array}\right.
$$

In order to simplify the analysis we enclose our system in a spherical box of radius $R \gg m$, and impose the condition that the field vanishes at the boundary, in this way the energy spectrum becomes discrete. By using the gauge constraints and equating the terms of the same order $\frac{\hbar}{i}$ we have

$$
\left(\begin{array}{c}
H(r) \\
K(r) \\
G_{1}(r) \\
G_{2}(r) \\
U(r) \\
V(r)
\end{array}\right)=\left(\begin{array}{c}
\left(\frac{\hbar}{i}\right)^{2} h_{2}(r) \\
\left(\frac{\hbar}{i}\right)\left[1-\frac{l(l+1)}{2}\right] \frac{g_{2}(r)}{\dot{s}_{0}(r)} \frac{1}{N^{2} r^{2}}+\left(\frac{\hbar}{i}\right)^{2} \mathcal{H}_{2}(r) \\
\frac{l(l+1)}{2} g_{20}(r)+\left(\frac{\hbar}{i}\right) \frac{l(l+1)}{2} g_{21}(r)+\left(\frac{\hbar}{i}\right)^{2}\left[\frac{l(l+1)}{2} g_{2_{2}}(r)-h_{2}(r)\right] \\
g_{20}(r)+\left(\frac{\hbar}{i}\right) g_{21}(r)+\left(\frac{\hbar}{i}\right)^{2} g_{2_{2}}(r) \\
u_{0}(r)+\left(\frac{\hbar}{i}\right) u_{1}(r)+\left(\frac{\hbar}{i}\right)^{2} u_{2}(r) \\
v_{0}(r)+\left(\frac{\hbar}{i}\right)^{2} v_{1}(r)+\left(\frac{\hbar}{i}\right)^{2} v_{2}(r) \\
e^{\frac{i}{\hbar}\left(s_{0}(r)+\left(\frac{\hbar}{i}\right) s_{1}(r)+\left(\frac{\hbar}{i}\right)^{2} s_{2}(r)\right)},
\end{array}\right)
$$

with the conditions $\left(\dot{f}=\frac{d}{d r} f(r)\right)$ :

$$
\begin{aligned}
& h_{2}(r)=l(l+1) \frac{k_{1}(r)}{\dot{s}_{0}(r)} \\
& k_{2}(r)=-\frac{1}{\dot{s}_{0}(r)}\left[\dot{\mathcal{H}}_{1}(r)+\frac{4}{r} k_{1}(r)+g_{21}(r)\left(1-\frac{l(l+1)}{2}\right)+k_{1} \dot{s}_{1}(r)\right] \\
& k_{1}(r)=\left[1-\frac{l(l+1)}{2}\right] \frac{g_{2}}{\dot{s}_{0}(r)} \frac{1}{N^{2} r^{2}} .
\end{aligned}
$$

Inserting these expressions in the eigenvalue equations (79), we can check that the following is a solution:

$$
\left(\begin{array}{c}
H(r) \\
K(r) \\
G_{1}(r) \\
G_{2}(r) \\
U(r) \\
V(r)
\end{array}\right)=\left(\begin{array}{c} 
\pm\left(\frac{\hbar}{i}\right)^{2} l(l+1)\left[1-\frac{l(l+1)}{2}\right] \frac{N^{2}}{4 \lambda r^{2}} \\
\pm\left(\frac{\hbar}{i}\right)\left[1-\frac{l(l+1)}{2}\right] \frac{1}{2 \sqrt{\lambda} r^{2}}-\left(\frac{\hbar}{i}\right)^{2}\left[1-\frac{l(l+1)}{2}\right] \frac{1}{4 \lambda r^{3}} \\
\frac{l(l+1)}{2} \mp\left(\frac{\hbar}{i}\right)^{2} l(l+1)\left[1-\frac{l(l+1)}{2}\right] \frac{N^{2}}{4 \lambda r^{2}} \\
1 \\
0 \\
0
\end{array}\right)
$$




$$
\cdot N^{2} \frac{c}{r} e^{ \pm \frac{i}{\hbar} 2 \sqrt{\lambda} r^{*}+\alpha(l)+O(\hbar)}
$$

where $c$ is an integration constant, depending in general on $l$, and $r^{*}=r+$ $2 m \log \left(\frac{r}{2 m}-1\right)$. The constant $\alpha(l)$ is crucial for determining the eigenvalues. As illustrated in Appendix E, $\alpha(l)$ can be determined by comparing (101) with the exact solutions for the flat case $M=0$, obtaining

$$
\alpha(l)=-i(l+1) \frac{\pi}{2} .
$$

We shall now determine the regime of validity of our approximation. We observe that (101) has been obtained by expanding the eigenvalue equations up to the order $\left(\frac{\hbar}{i}\right)$. By considering the next order one gets:

$$
s_{2}(r)= \pm \frac{1}{4 \sqrt{\lambda}}\left[\frac{1}{r}+\frac{1}{2 m} \ln \frac{r}{r-2 m}\right] .
$$

so that $\hbar\left|s_{2}(r)\right| \ll 1$ if $2 \frac{\sqrt{\lambda} r}{\hbar} \gg 1$, implying that, as expected, the approximation is good in the very high energy region. Before imposing the boundary conditions, let us analyse the behaviour of the "odd" part.

Looking for a solution of the form:

$$
\begin{aligned}
\left(\begin{array}{c}
F_{1}(r) \\
F_{2}(r) \\
D(r)
\end{array}\right)= & \left(\begin{array}{c}
L(r) \\
M(r) \\
d(r)
\end{array}\right) e^{\frac{i}{\hbar} A(r)} \\
= & \left(\begin{array}{c}
L_{0}(r)+\frac{\hbar}{i} L_{1}(r)+\left(\frac{\hbar}{i}\right)^{2} L_{2}(r) \\
M_{0}(r)+\frac{\hbar}{i} M_{1}(r)+\left(\frac{\hbar}{i}\right)^{2} M_{2}(r) \\
d_{0}(r)+\frac{\hbar}{i} d_{1}(r)+\left(\frac{\hbar}{i}\right)^{2} d_{2}(r)
\end{array}\right) \\
& \cdot e^{\frac{i}{\hbar} A_{0}(r)+A_{1}(r)+\frac{\hbar}{i} A_{2}(r)}
\end{aligned}
$$

and setting $M_{0}=1$ and $M_{j}=0(j>1)$, we easily find the solution:

$$
\begin{aligned}
\left(\begin{array}{c}
F_{1}(r) \\
F_{2}(r) \\
D(r)
\end{array}\right)= & \left(\begin{array}{c} 
\pm\left(\frac{\hbar}{i}\right)\left[1-\frac{l(l+1)}{2}\right] \frac{1}{2 \sqrt{\lambda} r^{2}}-\left(\frac{\hbar}{i}\right)^{2}\left[1-\frac{l(l+1)}{2}\right] \frac{N^{2}}{4 \lambda r^{3}} \\
0 \\
0
\end{array}\right) \\
& \cdot N^{2} \frac{c}{r} e^{ \pm \frac{i}{\hbar} 2 \sqrt{\lambda} r^{*}-i(l+1) \frac{\pi}{2}+O(\hbar)} .
\end{aligned}
$$

Let us first consider the flat case $M=0$. By imposing the vanishing of the solutions, both "even" and "odd" for $r=R$, we obtain the spectrum:

$$
2 \frac{\sqrt{\lambda_{n, l}^{0}}}{\hbar} R-l(l+1) \frac{\pi}{2}=n \frac{\pi}{2}
$$


or

$$
\sqrt{\lambda_{n, l}^{0}}=\frac{\hbar}{2 R}(n+l+1) \frac{\pi}{2}
$$

For $M \neq 0$, one gets instead:

$$
\sqrt{\lambda_{n, l}}=\sqrt{\lambda_{n, l}^{0}} \frac{R}{R^{*}}=\sqrt{\lambda_{n, l}^{0}} \frac{1}{1+\frac{2 m}{R} \ell \mathrm{n}\left(\frac{R}{2 m}-1\right)},
$$

which clearly shows that, due to that presence of the wormhole, the gravitons' spectrum is redshifted by the quantity $\frac{R}{R^{*}}=\frac{1}{1+\frac{2 m}{R} \ell \mathrm{n}\left(\frac{R}{2 m}-1\right)}$. In the next Section we shall analyse the consequences for the energy of our state of the results obtained so far.

\section{The energy of the quantized gravitational field around an ensemble of wormholes}

We have just seen that an external observer who looks into a finite spherical box (of radius $R$ ), centered around a wormhole, perceives that the gravitons contained in it are redshifted with respect to the gravitons in the absence of the wormhole. We wish now to determine the over all energy shift

$$
\Delta E(M)=E(M)-E(0)
$$

between the gravitational quantum state in the box containing a wormhole and the one with no wormhole. In Section 2 we have seen that

$$
E(M)=M+\sum_{n, l, m, \sigma} \sqrt{\lambda_{n, l, m}^{\sigma}}
$$

where $l, m$ are the angular quantum numbers, $n$ is the radial one and $\sigma=\mp$ for "even" or "odd" solutions. Please note that we have come back to the natural units where $\hbar=c=1$. In flat space-time the energy is

$$
E^{0}=\sum_{n, l, m, \sigma} \sqrt{\lambda_{n, l, m}^{0 \sigma}}
$$

Thus, from (105) and (106) we compute

$$
\Delta E(M)=E(M)-E(0) \simeq M-\frac{\pi \hbar}{2 R^{2}} m \log \frac{R}{2 m} \sum_{n, l, m, \sigma}(n+l+1) .
$$

We must now sum over the quantum numbers, we get

$$
\sum_{n, l, m, \sigma}(n+l+1)=\sum_{n l} 2(2 l+1)(n+l+1) .
$$


and introducing the momentum cut-off $\Lambda$ 目 we have,

$$
\Lambda=\frac{\pi}{4 R} N \geq \frac{\pi}{4 R}(n+l+1) .
$$

From:

$$
\begin{aligned}
& \sum_{n+l+1 \leq N} 2(2 l+1)(l+n+1)=\frac{N^{4}}{2}-\frac{17}{18} N^{3}+\frac{N}{9} \\
& \sim \frac{N^{4}}{2}=\frac{64 R^{4}}{(\hbar \pi)^{4}} \Lambda^{4} .
\end{aligned}
$$

we may finally write:

$$
\Delta E(M)=M-\frac{64 \Lambda^{4} R^{2}}{\pi^{3} \hbar^{3}} G M \log \frac{R}{2 G M},
$$

or equivalently for the energy density:

$$
\frac{\Delta E(M)}{V}=\frac{M}{\frac{4 \pi}{3} R^{3}}-\frac{36 \Lambda^{4}}{\pi^{4} \hbar^{3}}\left(\frac{G M}{R}\right) \ln \left(\frac{R}{2 G M}\right),
$$

which clearly shows that the "stable" gravitons' modes on the Schwarzschild background, due to the gravitational red-shift, have a zero-point energy smaller than in flat space-time, leading to the negative term in (113). However we shall not analyse (113) any further, for this expression is deficient in two important aspects: (i) it does not incorporate the (negative) classical contribution from the "unstable" mode (96); (ii) it only describes a single, isolated "wormhole" which, in view of the large energy gains involved, if certainly does not correctly describe what we are after: a possible QG Ground State. In any event, the importance of (113) lies in its exposing unequivocally the quantum mechanical instability of the classical Ground State for the matterless universe: the (pseudo) euclidean space-time.

\section{The multi-wormhole state}

In order to have a realistic model for the Ground State of QG, as we have just argued, we must seek a more realistic classical solution of matterless Einstein's gravity. It is clear from what we have learned so far that the natural candidate is a "condensed" system of WH's, either gaseous or crystalline, of ADM mass $M$ and (average) density $\left(\frac{1}{a}\right)^{3}$, a being the interwormhole average distance. Even though no explicit solution, that we know of, has been given for the general

\footnotetext{
${ }^{5}$ The problem of the cut-off, as already discussed, is a very important one. For consistency of our approach requires $G(\Lambda) \Lambda^{2} \leq 1$, where $G(\Lambda)$ is the value of the Newton constant at the cut-off $\Lambda$.
} 
case, one such classical state must certainly exist, for by increasing $a$ one is led to the well-known Schwarzschild solution, and special configurations of the $n$ wormhole system have been studied and characterized in ref. [11]. From this study one confirms the intuitive expectation that the ADM energy of "wormholes" of mass $M$, for separations $a \gg 2 G M$ (the Schwarzschild radius), coincides with the classical energy of a system of $n$ matter points interacting via the two-body Newtonian potential $U=-\frac{G M^{2}}{a}$. Classically this configuration is unstable, since under the action of the Newtonian attraction the "wormholes" will not keep their (average) mutual distances a but will tend to collapse to a single "wormhole" configuration, encompassing the given space-time $V$. Quantum -mechanically the situation is different, for the Heisenberg principle puts in general bounds upon the interwormhole distance $a$. Indeed, for a two-wormhole system, from the Schrödinger equation

$$
\left[-\frac{\hbar^{2}}{2 \mu} \nabla_{\vec{d}}^{2}+U(d)\right] u(\vec{d})=E u(\vec{d}),
$$

where the reduced mass $\mu=\frac{M}{2}$, we obtain for the lowest lying state:

$$
E=-\frac{G M^{2}}{2 a_{\circ}}
$$

with the "Bohr-radius"

$$
a_{\circ}=\frac{2}{G M^{3}} .
$$

Thus, provided the "Bohr radius" $a_{\circ}$ exceeds the "coalescence" distance $d_{c}=$ $4 G M$ (twice the Schwarzschild radius) the quantum mechanical system of $n$ wormholes is stable. This means that one must have:

$$
a \geq a_{\circ} \geq 4 G M
$$

the maximum allowed density occurring when

$$
a=a_{\circ}=4 G M,
$$

i.e. for

$$
M=\left(\frac{1}{2}\right)^{\frac{1}{4}} G^{-\frac{1}{2}}
$$

Extending (113) to the $n$ "wormholes" case, and setting $R=\frac{a}{2}$, we obtain for the energy density difference $\left(a \geq 4 G M=\frac{2}{G M^{3}}\right)$

$$
\frac{\Delta E}{V} \simeq \frac{M}{a^{3}}\left[1-\frac{G M}{4 a}-\frac{16}{\pi^{3}} G a^{2} \Lambda^{4} \ell \mathrm{n}\left(\frac{a}{4 G M}\right)\right]
$$

\footnotetext{
${ }^{6}$ Evidently part of the energy in the collapse must be radiated away, as the single wormhole mass in the volume $V$ is proportional to $n^{\frac{1}{3}} M$ and not to $n M$.
} 
Thus, the energy density gain is maximum when $a$ is minimum, i.e. when, according to (117)

$$
a=a_{\circ}=\frac{2}{G M^{3}} .
$$

On the other hand, the quantum stability of the $n$-wormhole configuration teaches us that the minimum value of the Bohr radius $a_{\circ}$ must equal $4 G M$, implying that the maximum energy gain is achieved when the wormholes have, according to (120), an ADM-mass $M=\left(\frac{1}{2}\right)^{\frac{1}{4}} G^{-\frac{1}{2}}=\left(\frac{1}{2}\right)^{\frac{1}{4}} m_{p}$, and an average distance

$$
a_{\circ}=\frac{2}{G \mu^{3}}=\left(\frac{1}{2}\right)^{\frac{7}{4}} G^{-\frac{1}{2}}=\left(\frac{1}{2}\right)^{\frac{7}{4}} l_{p} .
$$

We have just seen that, even without taking into account the (negative) contribution to the energy density of the unstable modes, a system of "wormholes" of ADM mass $M=2^{-\frac{1}{4}} m_{p}\left(m_{p}=G^{-\frac{1}{2}} \simeq 10^{19} \mathrm{GeV}\right.$, the Planck mass $)$ at the average distance $\left(\frac{1}{2}\right)^{\frac{7}{4}} l_{p}\left(l_{p}=G^{\frac{1}{2}} \simeq 10^{-33} \mathrm{~cm}\right.$, the Planck length) realize, according to (121), a large energy (density) gain with respect to the Perturbative Ground State (PGS) of Quantum Gravity.

We shall now try to answer the important question of the so far neglected contribution of the "unstable" mode around a single wormhole. Let us consider around a wormhole of mass $M$, of Schwarzschild radius $2 m=2 G M$, a spherical region of radius $R=\lambda m\left(\frac{a}{m}>\lambda>2\right)$. The spherical region shell: $2 m<r<\lambda m$ is the region (modulo the quantum fluctuations of the wormhole), which the asymptotic observer can probe, and in particular measure its (average) Riemann tensor.

The existence around the wormhole of an "unstable" solution of the gravitational field (which will obviously generalize to the case of many wormholes), found in section 3, guarantees us that such a mode will contribute a classical (negative) term to the ADM energy of the quantized gravitational field. What kind and size of contribution? As argued in Section 3, without involving ourselves in the intractable expansion of the QG Hamiltonian to 3rd and higher orders, we may figure out the effect of the "unstable" mode in the following way. Let us call $\phi_{i j}^{(u)}$ the normalized unstable mode, which according to the developments in section 4, can be expressed in terms of the function $H(r)$ given (94) and the constraint function $U(r)$, appearing in (96). Its contribution $h_{i j}(x)$ to the quantized gravitational field is thus

$$
h_{i j}^{u}=A \phi_{i j}^{u},
$$

where $A$ is a real constant whose value shall be determined in such a way as to minimize the Riemann tensor associated with the metric field

$$
g_{i j}=\eta_{i j}^{s}+h_{i j}^{u} .
$$


Thus we examine the metric:

$$
\begin{gathered}
g_{11}=\left(1-\frac{2 m}{r}\right)^{-1}-2 A \frac{1}{\sqrt{1-\frac{2 m}{r}}} r^{-3} \\
g_{22}=r^{2}+A \sqrt{1-\frac{2 m}{r}} r^{-1} \\
g_{33}=\left(r^{2}+A \sqrt{1-\frac{2 m}{r}} r^{-1}\right)(\sin \theta)^{2}
\end{gathered}
$$

which yields the non-vanishing components of the 3-dimensional Riemann tensor are:

$$
\begin{aligned}
& R_{121}^{2}=\left(123 m^{2} A^{2} r^{3}+12 m^{2} A^{3} \sqrt{-\frac{-r+2 m}{r}}-116 m A^{2} r^{4}-4 m r^{10}\right. \\
& \left.+14 r^{7} \sqrt{-\frac{-r+2 m}{r}} m A-8 m A^{3} r \sqrt{-\frac{-r+2 m}{r}}+27 A^{2} r^{5}\right) \\
& \left(4 r^{4} \sqrt{-\frac{-r+2 m}{r}} 8 A r+16 A m\right)^{-1} \frac{1}{\sqrt{-\frac{-r+2 m}{r}}} \\
& \left(r^{3}+A \sqrt{-\frac{-r+2 m}{r}}\right)^{-2} r^{-3} \\
& R_{232}{ }^{3}=\left(-12 r^{5} \sqrt{-\frac{-r+2 m}{r}} A m-9 A^{2} r^{3}+40 A^{2} r^{2} m-53 A^{2} m^{2} r\right. \\
& \left.+8 m r^{8}-16 m^{2} r^{7}+24 A m^{2} r^{4} \sqrt{-\frac{-r+2 m}{r}}+18 A^{2} m^{3}\right) 1 / 4 r^{-2} \\
& \left(r^{3}+A \sqrt{-\frac{-r+2 m}{r}}\right)^{-1} \frac{1}{\sqrt{-\frac{-r+2 m}{r}}} \\
& \left(r^{4} \sqrt{-\frac{-r+2 m}{r}}-2 A r+4 A m\right)^{-1}
\end{aligned}
$$

Averaging these expression in a spherical shell $2 m<r<3 m$, we have checked numerically that for $A \simeq 14(G M)^{3}$,

$$
\left|\frac{\left\langle R_{121}^{2}\right\rangle}{\left\langle R_{121}^{2(s)}\right\rangle}\right| \simeq\left|\frac{\left\langle R_{232}^{3}\right\rangle}{\left\langle R_{232}^{3(s)}\right\rangle}\right| \ll 1,
$$


clearly showing the possibility that in a shell of radius $O(m)$ around a "wormhole" the "unstable" mode screens completely the classical curvature induced by the "wormhole" itself. In view of the general result that the zero-curvature metric realizes the minimum energy density of the classical gravitational field, the maximum screening of the curvature existing around of a "wormhole" allows the "unstable" mode of the quantized gravitational field to produce the minimization of the ADM (classical) energy that characterizes the Ground State of Quantum Gravity.

\section{The gas of wormholes: a possible ground state of quantum gravity}

Let us now, in this concluding Section, try to distill the main points and results of this work. In order to have a better understanding of what has been achieved, let us first describe again the strategy we have adopted to probe the stability of the classical ground state - the flat Minkowskian background - subject to the fluctuations of the quantized gravitational field.

For definiteness' sake, for the classical solution of matterless gravity, our Ansatz for the classical background of QG, we have chosen the Schwarzschild solution but other possible starting points could be the Reissner-Nordstrom or the Kerr solution. From the classical standpoint such a background cannot be a good model for the vacuum, for it is well-known that the ADM[7] mass of such solution is positive $\left(E_{A D M}=M\right)$, whereas $E_{A D M}=0$ for flat space-time. But for the quantized gravitational field things may in principle be quite different, for $E_{A D M}$ receives contributions not only from the classical background field but also from the quantum fluctuations described by the quantum field $h_{i j}$ related to the metric field by:

$$
g_{i j}=\eta_{i j}^{(s)}+h_{i j}
$$

$\eta_{i j}^{(s)}$ being the Schwarzschild solution, fully characterized by the ADM-mass $M$ (and Schwarzschild radius $r_{s}=2 G M=2 m$ ). And it may happen, as it has been discovered in ref.[2], that the full energy $E(M)$ classical plus quantum, of the configuration (129) may turn out to be smaller than quantum energy $E(0)$ of the Perturbative Ground State (PGS), where $\eta_{i j}^{s} \rightarrow \delta_{i j}$. If this indeed happened one would have finally got rid of the embarrassing PGS and of the unrenormalizable perturbative field theory that inevitably obtains upon it.

As stressed in the Introduction, our hope that such strategy might lead to a similar situation, thus giving us a sensible QG, was based on a similar analysis performed on a non-abelian gauge theory, the $S U(2)$ pure Yang-Mills theory (akin to QCD) [2], where it was discovered that the energy $E(B)$, of the quantized field fluctuating around a classical solution, describing a constant chromomagnetic field $B$, was in fact lower than the PGS-energy $E(0)$. In the course of this analysis 
it was also discovered that around this non-trivial classical background there exist "unstable" modes (whose frequencies of small oscillations are imaginary) that profoundly modify the structure of the theory even at very high frequencies, fatally undermining the paradigmatic Asymptotic Freedom [12]. Of course, our hope was strengthened by the observation that QG may be looked at as a nonabelian gauge theory whose group is the Poincaré group, acting upon the tangent Minkowskian spaces.

The analysis reported in Sections 4 and 5 reveals that around an isolated "wormhole":

1. there exist an S-wave "unstable" mode [see (94), (96) and (97)];

2. the high energy "stable" modes are red-shifted with respect to the gravitons of flat Euclidean space, and realize the large energy gain $\Delta E(M)$ of eq.(113).

As a result the PGS is clearly seen to be unstable: the quantized field finds it energetically advantageous to "concentrate" in a "wormhole" of radius $2 G M$ and "mass" $M$, which redshifts the zero-point modes of the gravitational field, lowering in this way their energy density.

But, as shown in Section 6, the single "wormhole" configuration certainly does not minimize the energy of the gravitational field, for the appearance of several other "wormholes" of mass $M$ at an appropriate inter-wormholes (average) distance $a$ - a gas or a lattice of "wormholes" - leads to a decrease of the energy density [see eq.(121)] provided, however, that $a$ is bigger than the distance $d=$ $4 G M$, at which two of them coalesce into a single "wormhole". Taking into account such constraint, from (121) and (122) we derive that an ensemble of "wormholes" with mass $M \sim G^{-\frac{1}{2}}=m_{p}$ at the (average) distance $a \simeq G^{\frac{1}{2}}=l_{p}$ minimizes the part of the energy density that takes no account of the (negative) classical energy density stemming from the so far neglected "unstable" modes.

At the end of section 6 by a numerical analysis we show that the variational amplitude $A$ of the "unstable" mode [see eq.(124)] can be chosen in such a way that the classical part of the Riemann tensor, averaged over a spherical shell $(2 m<r<3 m)$ around a "wormhole" is much smaller than the averaged Riemann tensor of the Schwarzschild solution. In this way the energy density can be further lowered, obtaining a state of the quantized gravitational field, fluctuating around a gas of wormholes of Planck mass with Planck distance separation, whose energy density is way below the energy density of the PGS: an excellent candidate for the GS of QG.

Why do we believe that this latter statement is of significance for our understanding of the fundamental laws of physics? First of all we must note, as emphasized in the Introduction, that the gas of "wormholes" that minimizes the energy density of the quantized gravitational field realizes the remarkable intuition of J.A. Wheeler that at the Planck distance continuous space may dissolves 
in a kind of foam whose voids have the Planck size $l_{p}$. Even though Wheeler's foam can only be seen when our space resolution reaches $l_{p}$, just an impossible dream for presently available "microscopes" (the high energy accelerators), its effect on the structure and self-consistency of the QFT's of the Standard Model and for QG itself is enormous. In fact the space-structure upon which the observable quantum fields are defined turns out to be essentially discrete, comprising the Planck size space domains located at the interstices of the Planck size WH's which form the "points" of a "Planck Lattice" (PL).

This latter statement might appear somewhat surprising, in view of the fact that the idea of a "foam" seems to retain a basic element of continuity of spacetime which is lost in a lattice formulation. However, if we now look at the physics of the phenomenon of "WH-condensation" which, we have shown, leads possibly to the real GS of QG, we realize at once that no meaning can be attached to the value of the gravitational field or, for that matter, of any other physical field, in space-time domains whose (linear) size is less than the Planck length $l_{p}$. And this for two reasons, first domains of such size are hidden beyond the horizen of the single WH and cannot affect the physics of the observable space-time domains (of the same size) apart from their gravitational effects, that are however compeltely screened by the unstable modes; and second the gas-like structure of the system of WH's with their fast quantum fluctuations cannot but "average" any "local" field over Planck-sized space-time domains, thus rendering unobservable, indeed unphysical, all field modes whose wave-lengths are smaller than $l_{p}$. Without such modes in the spectrum of any physical field it obviously follows that there is no way to probe space-time deeper than $l_{p}$, thus allowing us to effective describe space-time as an essentially discrete structure, a random lattice of average lattice constant $a=l_{p}$.

Even without going into the details of the significant advances in a fundamental understanding of the SM that a research program based on the PL achieves we can realize at once that the discretization of space in a PL introduces a fundamental momentum cut-off $\Lambda \simeq m_{p}$, the Planck mass, thus removing at a basic level all divergences of QFT's, which since their formulations at the end of the 20 's, have been a devastating conceptual difficulty, that the "conventionalistic" way out of "renormalization" could only throw "under the rug", arousing the anger of great minds such as Dirac's. With a PL, a faithful and adequate representation of the structure of the non-perturbative GS of QG, the ghosts of renormalization theory, such as the Landau's ghost, disappear beyond the horizon of the WH's, leaving us a decent perturbative expansion for the QFT's of the Standard Model, whose logarithmic divergences get converted to small corrections once one sets $\Lambda=m_{p}$. But this is not all, also the observable part of the quantized gravitational field, living on the PL, is cut -off at $m_{p}$, and the

\footnotetext{
${ }^{7} \mathrm{~A}$ concise bibliography is to be found in ref. 13 .

${ }^{8}$ This obviously does not happen for scalar theories, such as the Higgs model, where there appear "quadratic" divergences in $\Lambda^{2}$, which retain their devastating character, thus exposing
} 
"nonrenormalizable" divergences $O\left[\left(G \Lambda^{2}\right)^{n}\right]$ become small corrections once the providential powers of $2 \pi$ in the denominators, stemming from momentum integrations, are fully taken into account 9 . In this way, QG leaves the "limbo" of the theories of uncertain "self-consistency" to assume the status of a perfectly well defined QFT, with a well-defined perturbative S-matrix, whose quantum effects are governed in a well-defined fashion by a very small coupling constant $G$, the Newton constant. And in view of all this one needs only the "Ockham's razor" to cut away much of the sophisticated theoretical developments, based on "superstrings", of the last 15 years.

Let us end this paper by asking ourselves whether there remain open problems. Let us emphasize that we deem our results qualitatively robust, especially as for as the derivation of a quantum foam is concerned; an interesting open problem is then certainly the extension of the analysis to more complicated vacuum solutions, such as the Reissner-Nordstrom and Kerr metric. A more refined analysis, aimed at determining the detailed structure of the quantum foam and its approximation as a PL, is certainly needed. We hope to come back to this problem in the near future. Finally we think that "vexata quaestio" of the "cosmological constant" $\Lambda_{c}$ may have an answer within the framework of this paper, and that the "unstable" quantized modes of the gravitational field around each WH may have a lot to do with the surprising, and extremely welcome, negligible value that $\Lambda_{c}$ has in our Universe.

We acknowledge the collaboration of S. Cacciatori and I. Spagnolatti in the early stage of this work.

\section{Appendix A: Christoffel Symbol and Ricci tensor for the background field}

We give detail calculations of some fundamental quantities generated by $\boldsymbol{\eta}$. We call

$$
\boldsymbol{\eta}=\left(\begin{array}{ccc}
\frac{1}{1-\frac{2 m}{r}} & 0 & 0 \\
0 & r^{2} & 0 \\
0 & 0 & r^{2} \sin ^{2} \theta
\end{array}\right)
$$

For more convenience, we select the cartesiane coordinate: $x^{1}=r \sin \theta \cos \varphi, x^{2}=$ $r \sin \theta \sin \varphi, x^{3}=r \cos \theta$, and

$$
d \vec{x}=\left(\frac{x^{1}}{r} d r+x^{3} \cos \varphi d \theta-x^{2} d \varphi ; \frac{x^{2}}{r} d r+x^{3} \sin \varphi d \theta+x^{1} d \theta ; \frac{x^{3}}{r} d r-r \sin \theta d \theta\right)
$$

their unplausibility.

${ }^{9}$ As we have been emphasizing in this work, the cut-off at $m_{p}$ fully justifies our neglect in the Hamiltonian of terms of order higher than two. 
and then

$$
d s^{2}=\left\{\left(1-\frac{2 m}{r}\right)^{-1}-1\right\} \frac{(\vec{x} \cdot d \vec{x})^{2}}{r^{2}}-(d \vec{x})^{2}
$$

where

$$
\vec{x} \cdot d \vec{x}=\sum_{a} x^{a} d x^{a} .
$$

As a result,

$$
\eta_{a b}=\delta_{a b}+\frac{x_{a} x_{b}}{r^{2}}\left(\frac{1}{1-\frac{2 m}{r}}-1\right)
$$

and, in particular, we obtain

$$
x_{r}=g_{r s} x^{s}=x^{r}\left(1-\frac{2 m}{r}\right) .
$$

We first calculate the Christoffel symbol,

$$
\Gamma_{a b}^{r(s)}=\frac{1}{2} \eta^{r s}\left(\partial_{a} \eta_{s b}+\partial_{b} \eta_{s a}-\partial_{s} \eta_{a b}\right) .
$$

Since

$$
\partial_{a} \eta_{s b}=\frac{2 m}{r^{3}\left(1-\frac{2 m}{r}\right)}\left(\delta_{a s} x_{b}+\delta_{a b} x_{s}\right)-\frac{x_{b} x_{s} 2 m}{r^{b}\left(1-\frac{2 m}{r}\right)^{2}}\left(3 r^{2}-4 m r\right) \frac{x_{a}}{r},
$$

and obtain,

$$
\begin{aligned}
\Gamma_{a b}^{r(s)}= & \frac{2 m}{r^{3}} \frac{\delta_{a b} x^{r}}{\left(1-\frac{2 m}{r}\right)}\left[\frac{3}{1-\frac{2 m}{r}}-\frac{4 m}{r\left(\frac{2 m}{r}\right)}\right] \\
& =\frac{2 m}{r^{3}} \frac{\delta_{a b} x^{a}}{1-\frac{2 m}{r}}-\frac{m x_{a} x_{b} x^{r}}{r^{5}\left(1-\frac{2 m}{r}\right)}\left[3+\frac{2 m}{r\left(1-\frac{2 m}{r}\right)}\right] .
\end{aligned}
$$

In the coordinate $(r, \theta, \varphi)$ we have:

$$
\begin{gathered}
\Gamma_{l m}^{1}=\left(\begin{array}{ccc}
\frac{-m}{r(r-2 m)} & 0 & 0 \\
0 & -(r-2 m) & 0 \\
0 & 0 & -(r-2 m) \sin ^{2} \theta
\end{array}\right) \\
\Gamma_{l m}^{2}=\left(\begin{array}{ccc}
0 & \frac{1}{r} & 0 \\
\frac{1}{r} & 0 & 0 \\
0 & 0 & -\sin \theta \cos \theta
\end{array}\right) \\
\Gamma_{l m}^{3}=\left(\begin{array}{ccc}
0 & 0 & \frac{1}{r} \\
0 & 0 & \operatorname{cotg} \theta \\
\frac{1}{r} & \operatorname{cotg} \theta & 0
\end{array}\right)
\end{gathered}
$$


We can then calculate the Ricci tensor:

$$
R_{a b}^{(s)}=\Gamma_{r a, b}^{r(s)}-\Gamma_{a b, r}^{r(s)}+\Gamma_{s a}^{r(s)} \Gamma_{r b}^{s(s)}-\Gamma_{a b}^{r(s)} \Gamma_{r s}^{r(s)} .
$$

Since

we find:

$$
\Gamma_{r a}^{r(s)}=-\frac{m}{r^{3}} \frac{x_{a}}{1-\frac{2 m}{r}},
$$

$$
\Gamma_{r a, b}^{r}=-\frac{m \delta_{a b}}{r^{3}\left(1-\frac{2 m}{r}\right)}+\frac{m x_{a} x_{b}}{r^{5}\left(1-\frac{2 m}{r}\right)^{2}}\left(3 \frac{4 m}{r}\right) .
$$

and

$$
\begin{aligned}
\Gamma_{a b, s}^{r(s)}= & \frac{2 m}{r^{3}} \delta_{a b} \delta_{r s}-\frac{6 m}{r^{5}} \delta_{a b} x_{r} x_{s}-\frac{m}{r^{5}}\left[3+\frac{2 m}{r\left(1-\frac{2 m}{r}\right)}\right]\left(\delta_{a s} x_{b} x_{r}\right. \\
& \left.+\delta_{s b} x_{a} x_{r}+\delta_{s r} x_{a} x_{b}\right)+\frac{m x_{a} x_{b} x_{r} x_{s}}{r^{7}}\left[15+\frac{6 r^{5}-10 m r^{4}}{r^{5}\left(1-\frac{2 m}{r}\right)^{2}}\right]
\end{aligned}
$$

From this equation, in particular we obtain:

$$
\begin{aligned}
\Gamma_{a b, r}^{r(s)}= & -5 x_{a} x_{b} \frac{m}{r^{5}}\left[3+\frac{2 m}{r\left(1-\frac{2 m}{r}\right)}\right]+\frac{m x_{a} x_{b}}{r^{5}}\left[15+\frac{6 r-10 m}{r\left(1-\frac{2 m}{r}\right)^{2}}\right] \\
= & \frac{2 m^{2} x_{a} x_{b}}{r^{6}\left(1-\frac{2 m}{r}\right)^{2}},
\end{aligned}
$$

and

$$
\begin{aligned}
\Gamma_{s a}^{r(s)} \Gamma_{r b}^{s(s)}= & \left\{\frac{2 m}{r^{3}} \delta_{s a} x_{r}-\frac{m x_{a} x_{s} x_{r}}{r^{5}}\left[3+\frac{2 m}{r\left(1-\frac{2 m}{r}\right)}\right]\right\} \\
& \cdot\left\{\frac{2 m}{r^{3}} \delta_{r b} x_{b}-\frac{m x_{r} x_{b} x_{s}}{r^{5}}\left[3+\frac{2 m}{r\left(1-\frac{2 m}{r}\right)}\right]\right\} \\
& =x_{a} x_{b} \frac{m^{2}}{r^{6}}\left[1+\frac{2 m}{r\left(1-\frac{2 m}{r}\right)}\right]^{2} \\
& =x_{a} x_{b} \frac{m^{2}}{r^{6}} \frac{1}{\left(1-\frac{2 m}{r}\right)^{2}} .
\end{aligned}
$$

At the end, we have

$$
-\Gamma_{a b}^{a(s)} \Gamma_{r s}^{s(s)}=\left\{\frac{2 m}{r} \delta_{a g} x_{r}-\frac{m x_{a} x_{b} x_{r}}{r^{5}}\left[3+\frac{2 m}{r\left(1-\frac{2 m}{r}\right)}\right]\right\}
$$




$$
\begin{gathered}
\frac{m}{r^{3}} \frac{x_{a}}{1-\frac{2 m}{r}}=\frac{2 m^{2}}{r^{4}} \frac{\delta_{a b}}{\left(1-\frac{2 m}{r}\right)} \\
-\frac{m^{2}}{r^{6}} \frac{x_{a} x_{b}}{\left(1-\frac{2 m}{r}\right)}\left[3+\frac{2 m}{r\left(1-\frac{2 m}{r}\right)}\right],
\end{gathered}
$$

and as a result:

$$
\begin{aligned}
R_{a b}^{(s)}= & -\frac{m \delta_{a b}}{r^{3}\left(1-\frac{2 m}{r}\right)}-\frac{4 m^{2} x_{a} x_{b}}{r^{6}\left(1-\frac{2 m}{r}\right)^{2}}+\frac{3 m x_{a} x_{b}}{r^{5}\left(1-\frac{2 m}{r}\right)^{2}} \\
& -\frac{2 m^{2} x_{a} x_{b}}{r^{6}\left(1-\frac{2 m}{r}\right)^{2}}+\frac{x_{a} x_{b} m^{2}}{r^{6}\left(1-\frac{2 m}{r}\right)^{2}}+\frac{2 m^{2}}{r^{4}} \frac{\delta_{a b}}{1-\frac{2 m}{r}} \\
& -\frac{m^{2}}{r^{6}} \frac{x_{a} x_{b}}{1-\frac{2 m}{r}}\left[2+\frac{1}{\left(1-\frac{2 m}{r}\right)}\right]=-\frac{m}{r^{3}} \delta_{a b}-\frac{2 m^{2}}{r^{6}} \frac{x_{a} x_{b}}{1-\frac{2 m}{r}} \\
& +\frac{3 m x_{a} x_{b}}{r^{5}\left(1-\frac{2 m}{r}\right)^{2}}=-\frac{m}{r^{3}} \eta_{a b}+\frac{3 m x_{a} x_{b}}{r^{5}\left(1-\frac{2 m}{r}\right)} .
\end{aligned}
$$

Owning to

$$
\frac{x_{a} x_{b}}{r^{2}} \frac{2 m}{1-\frac{2 m}{r}}=\eta_{a b}-\delta_{a b},
$$

the Ricci tensor of the background field is:

$$
R_{a b}^{(s)}=\frac{m}{r^{3}}\left(-\eta_{a b}+\frac{3 r}{2 m} \eta_{a b}-\frac{3 r}{2 m} \delta_{a b}\right) .
$$

Going back to the coordinate $(r, \theta, \varphi)$ in the matrix form, we have:

$$
R_{b}^{a(s)}=\left(\begin{array}{ccc}
-\frac{2 m}{r^{2}} & 0 & 0 \\
0 & \frac{m}{r^{2}} & 0 \\
0 & 0 & \frac{m}{r^{2}}
\end{array}\right) .
$$

In particular we find that the scalar of curvature is zero: $R^{(s)}=0$.

\section{Appendix B: Decomposing the Regge-Wheeler matrix}

Using the technique due to T.Regge and J.A. Wheeler [⿴囗十), we separate the angular part from radial part in the 3 -dimension tensors $\phi_{j}^{i}(r, \theta, \varphi)$, vector $v_{i}(r, \theta, \varphi)$ and scalar $\tau(r, \theta, \varphi)$.

The even part:

$$
\phi_{1}^{1}(r, \theta, \varphi)=H(r) Y_{l m}(\theta, \varphi),
$$




$$
\begin{gathered}
\phi_{2}^{2}(r, \theta, \varphi)=\left(G_{1}(r)+G_{2}(r) \partial_{\theta}^{2}\right) Y_{l m}(\theta, \varphi) \\
\phi_{3}^{3}(r, \theta, \varphi)=\left(G_{1}(r)+\frac{G_{2}(r)}{\sin ^{2} \theta} \partial_{\varphi}^{2}+G_{2}(r) \cot \theta \partial_{\theta}\right) Y_{l m}(\theta, \varphi) \\
\phi_{1}^{2}(r, \theta, \varphi)=K(r) \partial_{\theta} Y_{l m}(\theta, \varphi) \\
\phi_{1}^{3}(r, \theta, \varphi)=\frac{K(r)}{\sin ^{2} \theta} \partial_{\varphi} Y_{l m}(\theta, \varphi) \\
\phi_{2}^{3}(r, \theta, \varphi)=\frac{G_{2}(r)}{\sin ^{2} \theta}\left(\partial_{\theta}-\cot \theta\right) \partial_{\varphi} Y_{l m}(\theta, \varphi) \\
v_{1}(r, \theta, \varphi)=U(r) Y_{l m}(\theta, \varphi) \\
v_{2}(r, \theta, \varphi)=V(r) \partial_{\theta} Y_{l m}(\theta, \varphi) \\
v_{3}(r, \theta, \varphi)=V(r) \partial_{\varphi} Y_{l m}(\theta, \varphi) \\
\tau(r, \theta, \varphi)=T(r) Y_{l m}(\theta, \varphi) .
\end{gathered}
$$

The odd part:

$$
\begin{gathered}
\phi_{1}^{1}(r, \theta, \varphi)=0 \\
\phi_{2}^{2}(r, \theta, \varphi)=F_{2}(r) \frac{1}{\sin \theta}\left(\partial_{\theta}-\cot \theta\right) \partial_{\varphi} Y_{l m}(\theta, \varphi) \\
\phi_{3}^{3}(r, \theta, \varphi)=-\phi_{2}^{2}(r, \theta, \varphi), \\
\phi_{1}^{2}(r, \theta, \varphi)=-\frac{F_{1}(r)}{\sin \theta} \partial_{\varphi} Y_{l m}(\theta, \varphi), \\
\phi_{1}^{3}(r, \theta, \varphi)=\frac{F_{1}(r)}{\sin \theta} \partial_{\theta} Y_{l m}(\theta, \varphi), \\
\phi_{2}^{3}(r, \theta, \varphi)=\frac{1}{2} \frac{F_{2}(r)}{\sin \theta}\left(\frac{1}{\sin ^{2} \theta} \partial_{\varphi}^{2}+\cot \theta \partial_{\theta}-\partial_{\theta}^{2}\right) Y_{l m}(\theta, \varphi), \\
v_{1}(r, \theta, \varphi)=0 \\
v_{2}(r, \theta, \varphi)=D(r)\left(-\frac{1}{\sin \theta}\right) \partial_{\theta} Y_{l m}(\theta, \varphi) \\
v_{3}(r, \theta, \varphi)=D(r) \sin \theta \partial_{\theta} Y_{l m}(\theta, \varphi) .
\end{gathered}
$$

Obviously, the scalar has no odd part. 


\section{Appendix C: Explicit form of the eigenequa- tions of Hamiltonia}

We give the great details of calculations for obtaining the equations of Hamiltonia eigenfunctions and constrains.

Laplacians of tensors

We show how to operate the Laplaciano $\nabla^{2} \equiv \eta^{a b} \nabla_{a} \nabla_{b}$ where the derivatives are taken covariantly with respect to the matrix $\eta_{a b}$.

For a tensor with rank 2, we find:

$$
\begin{aligned}
\nabla^{2} h_{j}^{i} & =\eta^{a b} h_{j \mid a b}^{i}=\eta^{a b}\left[h_{j \mid a, b}^{i}+\Gamma_{r b}^{i} h_{j \mid a}^{r}-\Gamma_{j b}^{r} h_{r \mid a}^{i}-\Gamma_{a b}^{r} h_{j \mid r}^{i}\right] \\
& =\eta^{a b}\left[h_{j, a b}^{i}+\left(h_{j}^{r} \Gamma_{r a}^{i}-h_{r}^{i} \Gamma_{j a}^{r}\right)_{, b}+\Gamma_{r b}^{i} h_{j, a}^{r}+\Gamma_{r b}^{i} \Gamma_{s a}^{r} h_{j}^{s}-\Gamma_{r b}^{i} \Gamma_{j a}^{s} h_{s}^{r}\right. \\
& \left.-\Gamma_{j b}^{r} \Gamma_{s a}^{i} h_{r}^{s}-\Gamma_{j b}^{r} h_{r, a}^{i}+\Gamma_{j b}^{r} \Gamma_{r a}^{s} h_{s}^{i}-\Gamma_{a b}^{r} h_{j, r}^{i}-\Gamma_{a b}^{r} \Gamma_{s r}^{i} h_{j}^{s}+\Gamma_{a b}^{r} \Gamma_{j r}^{s} h_{s}^{i}\right] \\
& =\eta^{a b}\left[h_{j, a b}^{i}+2 \Gamma_{r a}^{i} h_{j, b}^{r}-2 \Gamma_{j a}^{r} h_{r, b}^{i}+h_{j}^{r} \Gamma_{r a, b}^{i}-h_{r}^{i} \Gamma_{j a, b}^{r}+\Gamma_{r b}^{i} \Gamma_{s a}^{r} h_{j}^{s}\right. \\
& \left.-2 \Gamma_{r b}^{i} \Gamma_{j a}^{s} h_{s}^{r}+\Gamma_{j b}^{r} \Gamma_{r a}^{s} h_{s}^{i}-\Gamma_{a b}^{r} h_{j, r}^{i}-\Gamma_{a b}^{r} \Gamma_{s r}^{i} h_{j}^{s}+\Gamma_{a b}^{r} \Gamma_{j r}^{s} h_{s}^{i}\right] .
\end{aligned}
$$

For a covariant vector, we find:

$$
\begin{aligned}
\nabla^{2} B_{a} & =\eta^{b c} B_{a \mid b c}=\eta^{b c}\left(B_{a \mid b, c}-\Gamma_{a c}^{r} B_{r \mid b}-\Gamma_{b c}^{r} B_{a \mid r}\right) \\
& =\eta^{b c}\left[\left(B_{a, b}-\Gamma_{a b}^{s} B_{s}\right)_{, c}-\Gamma_{a c}^{r}\left(B_{r, b}-\Gamma_{r b}^{s} B_{s}\right)-\Gamma_{b c}^{r}\left(B_{a, r}-\Gamma_{a r}^{s} B_{s}\right)\right] \\
& =\eta^{b c}\left[B_{a, b c}-\Gamma_{a b}^{s} B_{s, c}-\Gamma_{a b, c}^{s} B_{s}-\Gamma_{a c}^{r} B_{r, b}+\Gamma_{a c}^{r} \Gamma_{r b}^{s} B_{s}-\Gamma_{b c}^{r} B_{a, r}\right. \\
& \left.+\Gamma_{b c}^{r} \Gamma_{a r}^{s} B_{s}\right] .
\end{aligned}
$$

Using, in the place of $\Gamma_{b c}^{a}$, the vectors calculated in Appendix A, We easily find:

$$
\begin{aligned}
\nabla^{2} \phi_{1}^{1}= & {\left[\frac{r-2 m}{r} \partial_{r}^{2}+\frac{1}{r^{2}} \partial_{\theta}^{2}+\frac{1}{r^{2} \sin ^{2} \theta} \partial_{\varphi}^{2}+\frac{2 r-3 m}{r^{2}} \partial_{r}\right.} \\
& \left.+\cot \theta \partial_{\theta}-4 \frac{r-2 m}{r^{3}}\right] \phi_{1}^{1}-\frac{4(r-2 m)}{r^{2}}\left(\partial_{\theta}+\cot \theta\right) \phi_{1}^{2} \\
& -\frac{4(r-2 m)}{r^{2}} \phi_{1}^{3}+2 \frac{r-2 m}{r^{3}} \phi_{2}^{2}+2 \frac{r-2 m}{r^{3}} \phi_{3}^{3} ; \\
\nabla^{2} \phi_{2}^{2}= & {\left[\frac{r-2 m}{r} \partial_{r}^{2}+\frac{1}{r^{2}} \partial_{\theta}^{2}+\frac{1}{r^{2} \sin ^{2} \theta} \partial_{\varphi}^{2}+\frac{2 r-3 m}{r^{2}} \partial_{r}+\cot \theta \partial_{\theta}\right.} \\
& \left.-2 \frac{r-2 m}{r^{3}}-\frac{2 \cot ^{2} \theta}{r^{2}}\right] \phi_{2}^{2}+\frac{4(r-2 m)}{r^{2}} \partial_{\theta} \phi_{1}^{2} \\
& -\frac{4}{r^{2}} \cot \theta \partial_{\varphi} \phi_{2}^{3}+2 \frac{r-2 m}{r^{3}} \phi_{1}^{1}+2 \frac{\cot ^{2} \theta}{r^{2}} \phi_{3}^{3}
\end{aligned}
$$




$$
\begin{aligned}
& \nabla^{2} \phi_{3}^{3}=\left[\frac{r-2 m}{r} \partial_{r}^{2}+\frac{1}{r^{2}} \partial_{\theta}^{2}+\frac{1}{r^{2} \sin ^{2} \theta} \partial_{\varphi}^{2}+\frac{2 r-3 m}{r^{2}} \partial_{r}+\cot \theta \partial_{\theta}\right. \\
& \left.-2 \frac{r-2 m}{r^{3}}-\frac{2 \cot ^{2} \theta}{r^{2}}\right] \phi_{3}^{3}+\frac{4(r-2 m)}{r^{2}} \partial_{\varphi} \phi_{1}^{3} \\
& +\frac{4 \cot \theta}{r^{2}} \partial_{\varphi} \phi_{2}^{3}+4 \cot \theta \frac{r-2 m}{r^{3}} \phi_{1}^{2} \\
& +2 \frac{r-2 m}{r^{3}} \phi_{1}^{1}+2 \frac{\operatorname{cotg}^{2} \theta}{r^{2}} \phi_{2}^{2} \text {; } \\
& \nabla^{2} \phi_{1}^{2}=\left[\frac{r-2 m}{r} \partial_{\varphi}^{2}+\frac{1}{r^{2}} \partial_{\theta}^{2}+\frac{1}{r^{2} \sin ^{2} \theta} \partial_{\varphi}^{2}+\frac{4 r-5 m}{r^{2}} \partial_{r}\right. \\
& \left.+\frac{\cot \theta}{r^{2}} \partial_{\theta}-\frac{3 r-9 m}{r^{3}}-\frac{\cot ^{2} \theta}{r^{2}}\right] \phi_{1}^{2}-\frac{2}{r^{3}} \partial_{\theta} \phi_{1}^{1} \\
& -2 \frac{\cot \theta}{r^{2}} \partial_{\varphi} \phi_{1}^{3}-\frac{2}{r^{3}} \partial_{\varphi} \phi_{2}^{3}-\frac{2}{r^{3}}\left(\cot \theta+\partial_{\theta}\right) \phi_{2}^{2}+\frac{2 \cot \theta}{r^{3}} \phi_{3}^{3} ; \\
& \nabla^{2} \phi_{1}^{3}=\left[\frac{r-2 m}{r} \partial_{r}^{2}+\frac{1}{r^{2}} \partial_{\theta}^{2}+\frac{1}{r^{2} \sin ^{2} \theta} \partial_{\varphi}^{2}+\frac{4 r-5 m}{r^{2}} \partial_{r}+\frac{3 \cot \theta}{r^{2}} \partial_{\theta}\right. \\
& \left.-4 \frac{r-9 m}{r^{3}}\right] \phi_{1}^{3}+\frac{2}{r^{3} \sin ^{2} \theta} \partial_{\varphi} \phi_{1}^{1}+\frac{2 \cot \theta}{r^{2} \sin ^{2} \theta} \partial_{\varphi} \phi_{1}^{2} \\
& -\frac{2}{r^{3} \sin ^{2} \theta} \partial_{\varphi} \phi_{3}^{3}-\frac{2}{r^{3}}\left(\partial_{\theta}+3 \cot \theta\right) \phi_{2}^{3} \text {; } \\
& \nabla^{2} \phi_{2}^{3}=\left[\frac{r-2 m}{r} \partial_{r}^{2}+\frac{1}{r^{2}} \partial_{\theta}^{2}+\frac{1}{r^{2} \sin ^{2} \theta} \partial_{\varphi}^{2}+\frac{2 r-3 m}{r^{2}} \partial_{r}+\frac{3 \cot \theta}{r^{2}} \partial_{\theta}\right. \\
& \left.-\frac{3 r-4 m}{r^{3}}-\frac{3 \cot ^{2} \theta}{r^{2}}\right] \phi_{2}^{3}+\frac{2(r-2 m)}{r^{2} \sin ^{2} \theta} \partial_{\varphi} \phi_{1}^{2} \\
& +\frac{2(r-2 m)}{r^{2}} \partial_{\theta} \phi_{1}^{3}+\frac{2 \cot \theta}{r^{2} \sin ^{2} \theta} \partial_{\varphi} \phi_{2}^{2}-\frac{2 \cot \theta}{r^{2} \sin ^{2} \theta} \partial_{\varphi} \phi_{3}^{3} .
\end{aligned}
$$

While for a vector:

$$
\begin{aligned}
\nabla^{2} v_{1}= & {\left[\frac{r-2 m}{r} \partial_{r}^{2}-\frac{\hat{L}^{2}}{r^{2}}+\frac{2 r-m}{r^{2}} \partial_{r}-\frac{2(r-2 m)}{r^{3}}\right] v_{1} } \\
& -\frac{2}{r^{3}}\left[\partial_{\theta}+\cot \theta\right] v_{2}-\frac{2}{r^{3} \sin ^{2} \theta} \partial_{\varphi} v_{3} \\
\nabla^{2} v_{2}= & {\left[\frac{r-2 m}{r} \partial_{r}^{2}-\frac{\hat{L}^{2}}{r^{2}}+\frac{m}{r^{2}} \partial_{r}-\frac{r-m}{r^{3}}-\frac{1}{r^{2}} \cot ^{2} \theta\right] v_{2} } \\
& +\frac{2(r-2 m)}{r^{2}} \partial_{\theta} v_{1}-\frac{2 \cot \theta}{r^{2} \sin ^{2} \theta} \partial_{\varphi} v_{3} ;
\end{aligned}
$$




$$
\begin{aligned}
\nabla^{2} v_{3}= & {\left[\frac{r-2 m}{r} \partial_{r}^{2}-\frac{\hat{L}^{2}}{r^{2}}+\frac{m}{r^{2}} \partial_{r}+\frac{m}{r^{3}}-\frac{2}{r^{2}} \cot \theta \partial_{\theta}\right] v_{3} } \\
& +\frac{2(r-2 m)}{r^{2}} \partial_{\varphi} v_{1}+\frac{2 \cot \theta}{r^{2}} \partial_{\varphi} v_{2} .
\end{aligned}
$$

Here with $\hat{L}$ we mean the usual operator of angular momentum expressed in polar coordinate. According to the decomposition of Regge-Wheeler given in the Appendix B, we obtain:

The even part

Substituting the even part in the Appendix B into eqs.(169) -...(177); taking into account the rules of commutation, we find:

$$
\begin{gathered}
{\left[-\hat{L}^{2}, \frac{1}{\sin \theta}\left(\partial_{\theta}-\cot \theta\right)\right] Y_{l m}(\theta, \varphi)=2 \frac{\cot \theta}{\sin \theta}\left[-\hat{L}^{2}-2 \partial_{\theta}^{2}\right] Y_{l m}(\theta, \varphi)} \\
+\frac{4}{\sin ^{3} \theta}\left[\left(\partial_{\theta}-\cot \theta\right) Y_{l m}(\theta, \varphi)\right], \\
{\left[-\hat{L}^{2}, \frac{1}{\sin \theta}\right] Y_{l m}(\theta, \varphi)=-2 \frac{\cot \theta}{\sin \theta} \partial_{\theta}^{2} Y_{l m}(\theta, \varphi)+\frac{1}{\sin ^{3} \theta} Y_{l m}(\theta, \varphi),} \\
{\left[-\hat{L}^{2}, \partial_{\theta}\right] Y_{l m}(\theta, \varphi)=\left(1+\cot ^{2} \theta\right) \partial_{\theta} Y_{l m}(\theta, \varphi)+2 \cos \theta \partial_{\varphi}^{2} \frac{Y_{l m}(\theta, \varphi)}{\sin ^{3} \theta},} \\
{\left[-\hat{L}^{2}, \sin \theta \partial_{\theta}\right] Y_{l m}(\theta, \varphi)=-2 \cot \theta \sin \theta l(l+1) Y_{l m}(\theta, \varphi) .}
\end{gathered}
$$

Defining,

$$
D_{l} \equiv N^{2} \partial_{r}^{2}+\frac{2 r-3 m}{r^{2}} \partial_{r}-\frac{l(l+1)}{r^{2}}
$$

we easily obtain:

$$
\begin{aligned}
\nabla^{2} \Phi_{1}^{1}= & \left\{\left[D_{l}-4 \frac{r-2 m}{r^{3}}\right] H(r)+4 \frac{r-2 m}{r^{2}} l(l+1) K(r)\right. \\
& \left.+2 \frac{r-2 m}{r^{3}}\left[2 G_{1}(r)-G_{2}(r) l(l+1)\right]\right\} Y_{l m}(\theta, \varphi), \\
\nabla^{2} \Phi_{2}^{2}=\left\{\left[D_{l}-2 \frac{r-2 m}{r^{3}}\right]\left[G_{1}(r)+G_{2}(r) \partial_{\theta}^{2}\right]\right. & +2 \frac{r-2 m}{r^{3}} H(r)+4 \frac{r-2 m}{r^{2}} K(r) \partial_{\theta}^{2} \\
+ & \left.2 \frac{G_{2}(r)}{r^{2}}\left[l(l+1)+2 \partial_{\theta}^{2}\right]\right\} Y_{l m}(\theta, \varphi), \\
\nabla^{2} \Phi_{3}^{3}= & \left\{\left[D_{l}-2 \frac{r-2 m}{r^{3}}\right]\left[G_{1}(r)-G_{2}(r)\left(l(l+1)+\partial_{\theta}^{2}\right)\right]\right. \\
+ & 4 \frac{r-2 m}{r^{2}} K(r)\left[\frac{1}{\sin ^{2} \theta} \partial_{\varphi}^{2}+\cot \theta \partial_{\theta}\right]+2 \frac{r-2 m}{r^{3}} H(r) \\
& \left.-\frac{2}{r^{2}} G_{2}(r)\left[l(l+1)+2 \partial_{\theta}^{2}\right]\right\} Y_{l m}(\theta, \varphi),
\end{aligned}
$$




$$
\left.\begin{array}{rl}
\nabla^{2} \Phi_{1}^{2}= & \left\{\left[D_{l}+2 \frac{r-m}{r^{2}} \partial_{r}-\frac{2 r-9 m}{r^{3}}\right] K(r)+\frac{2}{r^{3}} H(r)-\frac{2}{r^{3}} G_{1}(r)\right. \\
& \left.+\frac{2}{r^{3}}[l(l+1)+1] G_{2}(r)\right\} \partial_{\theta} Y_{l m}(\theta, \varphi), \\
\nabla^{2} \Phi_{1}^{3}= & \left\{\left[D_{l}+2 \frac{r-m}{r^{2}} \partial_{r}-\frac{2 r-9 m}{r^{3}}\right] K(r)+\frac{2}{r^{3}} H(r)-\frac{2}{r^{3}} G_{1}(r)\right. \\
+ & \left.\frac{2}{r^{3}}[l(l+1)+1] G_{2}(r)\right\} \frac{1}{\sin ^{2} \theta} \partial_{\varphi} Y_{l m}(\theta, \varphi), \\
\nabla^{2} \Phi_{2}^{3}= & \left\{\left[D_{l}+2 \frac{r+2 m}{r^{3}}\right] G_{2}(r)+4 \frac{r-2 m}{r^{2}} K(r)\right\} \frac{1}{\sin ^{2} \theta}\left(\partial_{\theta}\right. \\
& -\cot \theta) \partial_{\varphi} Y_{l m}(\theta, \varphi), \\
\nabla^{2} v_{1}= & \left\{\left[\frac{r-2 m}{r} \partial_{r}^{2}-\frac{l(l+1)}{r^{2}}+\frac{2 r-m}{r^{2}} \partial_{r}-2 \frac{r-2 m}{r^{3}}\right] U(r)\right. \\
& \left.+\frac{2}{r^{3}} l(l+1) V(r)\right\} \partial_{\theta} Y_{l m}(\theta, \varphi), \\
\nabla^{2} v_{3} & =\left\{\left[\frac{r-2 m}{r} \partial_{r}^{2}-\frac{l(l+1)}{r^{2}}+\frac{m}{r^{2}} \partial_{r}+\frac{m}{r^{3}}\right] C(r)\right. \\
\nabla^{2} v_{2} & =\left\{\frac{r-2 m}{r} \partial_{r}^{2}-\frac{l(l+1)}{r^{2}}+\frac{m}{r^{2}} \partial_{r}+\frac{m}{r^{3}}\right] C(r) \\
& \left.+2 \frac{r-2 m}{r^{2}} B(r)\right\} \partial_{\theta} Y_{l m}(\theta, \varphi),
\end{array}\right\}
$$

The Odd part

Using eqs.(178), (179), (180), (181) and the expressions of the odd part in the Appendix B, we obtain:

$$
\begin{gathered}
\nabla^{2} \Phi_{1}^{1}=0 \\
\nabla^{2} \Phi_{2}^{2}=- \\
\quad\left[\left(D_{l}+2 \frac{r+2 m}{r^{3}}\right) F_{2}(r)-4 \frac{r-2 m}{r^{2}} F_{1}(r)\right] \\
\frac{1}{\sin \theta}\left[\partial_{\theta}-\cot \theta\right] \partial_{\varphi} Y_{l m}(\theta, \varphi), \\
\nabla^{2} \Phi_{3}^{3}=-\left[\left(D_{l}+2 \frac{r+2 m}{r^{3}}\right) F_{2}(r)-4 \frac{r-2 m}{r^{2}} F_{1}(r)\right] \\
\frac{1}{\sin \theta}\left[\partial_{\theta}-\cot \theta\right] \partial_{\varphi} Y_{l m}(\theta, \varphi),
\end{gathered}
$$




$$
\begin{aligned}
& \nabla^{2} \Phi_{1}^{2}= {\left[\left(D_{l}+2 \frac{r-m}{r^{2}} \partial_{r}-\frac{2 r-9 m}{r^{3}}\right) F_{1}(r)-\frac{1}{r^{3}}[l(l+1)-2] F_{2}(r)\right] } \\
& \cdot {\left[-\frac{1}{\sin \theta} \partial_{\varphi} Y_{l m}(\theta, \varphi)\right] } \\
& \nabla^{2} \Phi_{1}^{3}= {\left[\left(D_{l}+2 \frac{r-m}{r^{2}} \partial_{r}-\frac{2 r-9 m}{r^{3}}\right) F_{1}(r)-\frac{1}{r^{3}}[l(l+1)-2] F_{2}(r)\right] } \\
& \cdot \frac{1}{\sin \theta} \partial_{\varphi} Y_{l m}(\theta, \varphi), \\
& \nabla^{2} \Phi_{2}^{3}= {\left[\left(D_{l}+2 \frac{r+2 m}{r^{3}}\right) F_{2}(r)-4 \frac{r-2 m}{r^{2}} F_{1}(r)\right] \frac{1}{2 \sin \theta} } \\
& \cdot\left[\frac{1}{\sin ^{2} \theta} \partial_{\varphi}^{2}+\cot \theta \partial_{\theta}-\partial_{\theta}^{2}\right] Y_{l m}(\theta, \varphi), \\
& \nabla^{2} v_{2}\left[\frac{r-2 m}{r} \partial_{r}^{2}-\frac{l(l+1)}{r^{2}}+\frac{m}{r^{2}} \partial_{r}+\frac{m}{r^{3}}\right] D(r)\left(-\frac{1}{\sin \theta} \partial_{\varphi} Y_{l m}(\theta, \varphi)\right) \\
& \nabla^{2} v_{3}\left[\frac{r-2 m}{r} \partial_{r}^{2}-\frac{l(l+1)}{r^{2}}+\frac{m}{r^{2}} \partial_{r}+\frac{m}{r^{3}}\right] D(r)\left(\frac{1}{\sin \theta} \partial_{\varphi} Y_{l m}(\theta, \varphi)\right)
\end{aligned}
$$

\section{Explicit form of eigenequations}

The eigenequations are given by:

$$
\begin{aligned}
& N^{2}\left[-\nabla^{2} \Phi_{a}^{b}+3 \delta_{1}^{s} \Phi_{a \mid s}^{b} \frac{m}{r^{2}}-\delta_{1}^{s} \Phi_{a s}{ }^{\mid b} \frac{m}{r^{2}}-\delta_{1}^{s} \Phi_{s \mid a}^{b} \frac{m}{r^{2}}+\Phi_{c}^{b} R_{a}^{c}\right. \\
& \left.\quad+\Phi_{a}^{c} R_{c}^{b}-\frac{8 m^{2}}{r^{3}(r-2 m)} \delta_{(a}^{1} h_{1}^{b)}\right]+v^{b}{ }_{\mid a}+v_{a}^{\mid b}+\eta_{a}^{b} \tau=4 \lambda \Phi_{a}^{b}
\end{aligned}
$$

where $\tau$ is the scalar function explicitly expressed in eq. (73). Analogous to the decomposition of Regge-Wheller, we separate the radial part from the angular part.

\subsection{Equations for the even part:}

- Equations of eigen-vectors.

Substituting the symbols of Christoffel and tensor of Ricci indicated in Appendix A into the explicit formulas of $\Phi_{a}^{b}$ and $v_{a}$ in Appendix B for the even part, and using the formula calculated in C.1, we calculate: 
- the equation for $H(a=b=1)$ :

$$
\begin{aligned}
& N^{2}\left\{\left[\frac{r-2 m}{r} \partial_{r}^{2}+2 \frac{r-2 m}{r^{2}} \partial_{r}-\frac{2}{3} \frac{6 r^{2}-27 m r+23 m^{2}}{r^{3}(r-2 m)}\right] H(r)\right. \\
& +4 \frac{r-2 m}{r^{2}} l(l+1) K(r) \\
& \left.+2 \frac{r-2 m}{r^{3}}\left[2 G_{1}(r)-l(l+1) G_{2}(r)\right]\right\} Y_{l m}(\theta, \varphi) \\
& =\left\{-H(r) 4 \lambda+\frac{4}{3}\left[\frac{r-2 m}{r} \partial_{r} U(r)-\frac{r-3 m}{r^{2}} U(r)+\frac{V(r)}{2} \frac{l(l+1)}{r^{2}}\right]\right. \\
& \left.+N^{2} \frac{l(l+1)}{r^{2}} H(r)\right\} Y_{l m}(\theta, \varphi) .
\end{aligned}
$$

- the equation for the combine function $2 G_{1}(r)-l(l+1) G_{2}(r)(a=b=2$ an then $a=b=3)$ :

$$
\begin{aligned}
& N^{2}\left\{[ \frac { r - 2 m } { r } \partial _ { r } ^ { 2 } + 2 \frac { r - 2 m } { r ^ { 2 } } \partial _ { r } - \frac { 2 } { r ^ { 2 } } ] \left(2 G_{1}(r)\right.\right. \\
& \left.-l(l+1) G_{2}(r)\right)+\frac{4}{3} \frac{3 r^{2}-12 m r+7 m^{2}}{r^{3}(r-2 m)} H(r) \\
& \left.\quad-4 \frac{2 r-3 m}{r^{2}} l(l+1) K(r)\right\} Y_{l m}(\theta, \varphi) \\
& =\left\{-4 \lambda\left[2 G_{1}(r)-l(l+1) G_{2}(r)\right]\right. \\
& +N^{2} \frac{l(l+1)}{r^{2}}\left[2 G_{1}(r)-l(l+1) G_{2}(r)\right] \\
& -\frac{4}{3}\left[\frac{r-2 m}{r} \partial_{r} U(r)-\frac{r-3 m}{r^{2}} U(r)\right. \\
& \left.\left.+\frac{1}{2} \frac{V(r)}{r^{2}} l(l+1)\right]\right\} Y_{l m}(\theta, \varphi) .
\end{aligned}
$$

- the equation for $G_{2}(r)(a=b=2$ an then $a=b=2)$ :

$$
\begin{aligned}
N^{2} & \left\{\left[\frac{r-2 m}{r} \partial_{r}^{2}+\frac{4 r-7 m}{r^{2}} \partial_{r}+\frac{2}{r^{2}}\right] G_{2}(r)+2 \frac{2 r-3 m}{r^{2}} K(r)\right\} \\
& \cdot\left[2 \partial_{\theta}^{2}+l(l+1)\right] Y_{l m}(\theta, \varphi) \\
= & \left\{-4 \lambda 2 G_{2}(r)+2 \frac{V(r)}{r^{2}}+N^{2} \frac{l(l+1)}{r^{2}} G_{2}(r)\right\} \\
& \cdot\left[2 \partial_{\theta}^{2}+l(l+1)\right] Y_{l m}(\theta, \varphi) .
\end{aligned}
$$

- the equation for $K(r)(b=3$ e $a=1)$ :

$$
N^{2}\left\{\left[\frac{r-2 m}{r} \partial_{r}^{2}+\frac{4 r-7 m}{r^{2}} \partial_{r}+\frac{2}{r^{2}}\right] G_{2}(r)+2 \frac{2 r-3 m}{r^{2}} K(r)\right\}
$$




$$
\begin{aligned}
\cdot & {\left[2 \partial_{\theta}^{2}+l(l+1)\right] \frac{1}{\sin ^{2} \theta} \partial_{\varphi} Y_{l m}(\theta, \varphi) } \\
= & \left\{-4 \lambda 2 G_{2}(r)+2 \frac{V(r)}{r^{2}}+N^{2} \frac{l(l+1)}{r^{2}} G_{2}(r)\right\} \\
& \cdot\left[2 \partial_{\theta}^{2}+l(l+1)\right] \frac{1}{\sin ^{2} \theta} \partial_{\varphi} Y_{l m}(\theta, \varphi) .
\end{aligned}
$$

- Finally for $b=3$ and $a=2$ we obtain the equation per $G_{2}$ :

$$
\begin{aligned}
N^{2}\{ & {\left[\frac{r-2 m}{r} \partial_{r}^{2}+2 \frac{r-2 m}{r^{2}} \partial_{r}-\frac{2}{r^{2}}\right]\left(2 G_{1}(r)-l(l+1) G_{2}(r)\right) } \\
& \left.+\frac{4}{3} \frac{3 r^{2}-12 m r+7 m^{2}}{r^{3}(r-2 m)} H(r)-4 \frac{2 r-3 m}{r^{2}} l(l+1) K(r)\right\} \\
& \cdot \frac{1}{\sin ^{2} \theta}\left(\partial_{\theta}-\cot \theta\right) \partial_{\varphi} Y_{l m}(\theta, \varphi) \\
= & \left\{-4 \lambda\left[2 G_{1}(r)-l(l+1) G_{2}(r)\right]+N^{2} \frac{l(l+1)}{r^{2}}\left[2 G_{1}(r)\right.\right. \\
& \left.-l(l+1) G_{2}(r)\right]-\frac{4}{3}\left[\frac{r-2 m}{r} \partial_{r} U(r)-\frac{r-3 m}{r^{2}} U(r)\right. \\
& \left.\left.+\frac{1}{2} \frac{V(r)}{r^{2}} l(l+1)\right]\right\} \frac{1}{\sin ^{2} \theta}\left(\partial_{\theta}-\cot \theta\right) \partial_{\varphi} Y_{l m}(\theta, \varphi) .
\end{aligned}
$$

From this equation, we get the eq. (79) for the radial part.

- Equations of constrain are,

$$
\Phi_{i}^{i}=0 \quad \text { and } \quad\left(\frac{\Phi_{j}^{i}}{N}\right)_{\mid i}=0
$$

The second equation corresponds to

$$
\Phi_{j \mid i}^{i}=\frac{m}{r(r-2 m)} \Phi_{j}^{1} .
$$

The traceless condition leads to:

$$
\left[2 G_{1}(r)-G_{2}(r) l(l+1)+H(r)\right] Y_{l m}(\theta, \varphi)
$$

which coincides with (80).

For imposing the condition of transversality, we use commutating rules shown in paragraph C.1.

- Imposing the condition (207) for $j=1$, one gets:

$$
\left[\partial_{r} H(l)+\frac{3 r-7 m}{r(r-2 m)} H(r)-K(r) l(l+1)\right] Y_{l m}(\theta, \varphi)=0
$$


- if $j=2$ one gets:

$$
\begin{gathered}
\left\{r(r-2 m) \partial_{r} K(r)+4(r-2 m) K(r)+G_{1}(r)\right. \\
\left.\quad+G_{2}(r)[1-l(l+1)]\right\} \partial_{\theta} Y_{l m}(\theta, \varphi)=0
\end{gathered}
$$

- Finally, if $j=3$, one gets:

$$
\begin{gathered}
\left\{r(r-2 m) \partial_{r} K(r)+4(r-2 m) K(r)+G_{1}(r)\right. \\
\left.+G_{2}(r)[1-l(l+1)]\right\} \partial_{\varphi} Y_{l m}(\theta, \varphi)=0
\end{gathered}
$$

and one verifies that the boundary conditions (76) and (75) are equivalent to:

$$
\begin{aligned}
& \left.\left\{r^{2}[H(r) U(r)+l(l+1) K(r) V(r)]\right\}\right|_{r=+\infty} \\
& \quad=\left.\left\{r^{2}[H(r) U(r)+l(l+1) K(r) V(r)]\right\}\right|_{r=2 m}=0, \\
& \quad\left\{\begin{array}{lc}
U(r) \rightarrow O\left(\frac{1}{r}\right) & r \rightarrow+\infty \\
V(r) \rightarrow O(1) . &
\end{array}\right.
\end{aligned}
$$

\subsection{Equations for the odd part}

- Eigenequation:

- Putting $a=b=1$, one gets identity $1 \equiv 1$.

- Putting $a=b=2$, one gets the equation for $F_{2}(r)$ :

$$
\begin{aligned}
N^{2} & {\left[\left(D_{l}-3 \frac{m}{r^{2}} \partial_{r}+\frac{2}{r^{2}}\right) F_{2}(r)\right.} \\
+ & \left.2 \frac{2 r-3 m}{r^{2}} F_{1}(r)\right] \frac{1}{\sin \theta} \\
& \cdot\left[\partial_{\theta}-\cot \theta\right] \partial_{\varphi} Y_{l m}(\theta, \varphi) \\
= & {\left[-4 \lambda F_{2}(r)+\frac{1}{r^{2}} D(r)\right] \frac{1}{\sin \theta}\left[\partial_{\theta}\right.} \\
& -\cot \theta] \partial_{\varphi} Y_{l m}(\theta, \varphi) .
\end{aligned}
$$

- Putting $a=b=3$ one gets equation (213).

- Putting $a=1$ and $b=2$ one gets:

$$
N^{2}\left[\left(D_{l}+2 \frac{r-2 m}{r^{2}} \partial_{r}-\frac{2}{r^{2}} \frac{r^{2}-5 m r+5 m^{2}}{r-2 m}\right) F_{1}(r)\right.
$$




$$
\begin{aligned}
& \left.+\frac{1}{r^{3}}(2-l(l+1)) F_{2}(r)\right]\left[-\frac{1}{\sin \theta} \partial_{\varphi} Y_{l m}(\theta, \varphi)\right] \\
= & {\left[-4 \lambda F_{1}(r)-\frac{1}{r^{2}}\left(\partial_{r}-\frac{2}{r}\right) D(r)\right] } \\
& \cdot\left[-\frac{1}{\sin \theta} \partial_{\varphi} Y_{l m}(\theta, \varphi)\right] .
\end{aligned}
$$

- Putting $a=1$ and $b=2$ one gets:

$$
\begin{aligned}
N^{2}[ & \left(D_{l}+2 \frac{r-2 m}{r^{2}} \partial_{r}-\frac{2}{r^{2}} \frac{r^{2}-5 m r+5 m^{2}}{r-2 m}\right) F_{1}(r) \\
& \left.+\frac{1}{r^{3}}(2-l(l+1)) F_{2}(r)\right]\left[\frac{1}{\sin \theta} \partial_{\theta} Y_{l m}(\theta, \varphi)\right] \\
= & {\left[-4 \lambda F_{1}(r)-\frac{1}{r^{2}}\left(\partial_{r}-\frac{2}{r}\right) D(r)\right] } \\
& {\left[\frac{1}{\sin \theta} \partial_{\theta} Y_{l m}(\theta, \varphi)\right] . }
\end{aligned}
$$

- Putting $a=2$ and $b=3$ one gets:

$$
\begin{aligned}
N^{2} & {\left[\left(D_{l}-3 \frac{m}{r^{2}} \partial_{r}+\frac{2}{r^{2}}\right) F_{2}(r)+2 \frac{2 r-3 m}{r^{2}} F_{1}(r)\right] \frac{1}{2 \sin \theta} } \\
\cdot & {\left[\frac{1}{\sin ^{2} \theta} \partial_{\varphi}^{2}+\cot \theta \partial_{\theta}-\partial_{\theta}^{2}\right] Y_{l m}(\theta, \varphi) } \\
= & {\left[-4 \lambda F_{2}(r)+\frac{1}{r^{2}} D(r)\right] \frac{1}{2 \sin \theta}\left[\frac{1}{\sin ^{2} \theta} \partial_{\varphi}^{2}\right.} \\
& \left.+\cot \theta \partial_{\theta}-\partial_{\theta}^{2}\right] Y_{l m}(\theta, \varphi) .
\end{aligned}
$$

These give (88), (87), which are used for the conditions of gauge. The traceless condition is automatically satisfied, while the (207) gives:

- Per $j=1$ identity $0 \equiv 0$.

- Per $j=2$ :

$$
\begin{aligned}
& \left\{-\partial_{r} F_{1}(r) r(r-2 m)-4(r-2 m) F_{1}(r)\right. \\
& \left.\quad+F_{2}(r)\left[1-\frac{l(l+1)}{2}\right]\right\} \frac{1}{\sin \theta} \partial_{\varphi} Y_{l m}(\theta, \varphi)=0 .
\end{aligned}
$$

- Per $j=3$ :

$$
\begin{aligned}
& \left\{\partial_{r} F_{1}(r) r(r-2 m)+4(r-2 m) F_{1}(r)\right. \\
& \left.\quad-F_{2}(r)\left[1-\frac{l(l+1)}{2}\right]\right\} \frac{1}{\sin \theta} \partial_{\varphi} Y_{l m}(\theta, \varphi)=0 .
\end{aligned}
$$


Finally the boundary conditions (76) e (75) become:

$$
\begin{aligned}
& {\left[l(l+1) r^{2} F_{1}(r) D(r)\right]_{r=+\infty}} \\
& \quad=\left[l(l+1) r^{2} F_{1}(r) D(r)\right]_{r=2 m}=0
\end{aligned}
$$

and

$$
D_{1}(r) \rightarrow O(1) \quad \text { per } \quad r \rightarrow+\infty
$$

\section{Appendix D: Normalization of eigenfunc- tions}

According to the scalar product (43), one needs to normalize the quantity:

$$
\begin{aligned}
Q= & \int_{\Sigma} d r d \theta d \varphi \frac{r^{2} \sin \theta}{\left(1-\frac{2 m}{r}\right)}\left[\Phi_{1}^{1^{*}}(r, \theta, \varphi) \Phi_{1}^{1}(r, \theta, \varphi)+\Phi_{2}^{2^{*}}(r, \theta, \varphi) \Phi_{2}^{2}(r, \theta, \varphi)\right. \\
& \Phi_{3}^{3^{*}}(r, \theta, \varphi) \Phi_{3}^{3}(r, \theta, \varphi)+2 r(r-2 m) \Phi_{1}^{2^{*}}(r, \theta, \varphi) \Phi_{1}^{2}(r, \theta, \varphi) \\
& +2 r(r-2 m) \sin ^{2} \theta \Phi_{1}^{3^{*}}(r, \theta, \varphi) \Phi_{1}^{3}(r, \theta, \varphi) \\
& \left.+2 \sin ^{2} \theta \Phi_{2}^{3^{*}}(r, \theta, \varphi) \Phi_{2}^{3}(r, \theta, \varphi)\right]
\end{aligned}
$$

We introduce:

$$
K=K(l) ; \quad H=H(r) ; \quad G_{1}=G_{1}(r) ; \quad G_{2}=G_{2}(r) ; \quad F_{1}=F_{1}(r)
$$

and

$$
F_{2}=F_{2}(r) ; Y=Y_{l m}(\theta, \varphi)
$$

We indicate,

$$
Y_{l m}(\theta, \varphi)=\frac{e^{i m \varphi}}{\sqrt{2 \pi}} \frac{1}{C_{l m}} P_{l}^{m}(\cos \theta)(-1)^{\frac{|m|-m}{2}}
$$

where

$$
\begin{gathered}
C_{l m}=\sqrt{\frac{2}{2 l+1} \frac{(l+m) !}{(l-m) !}}, \\
P_{l}^{m}(\cos \theta)=\sin ^{|m|} \theta \frac{d^{|m|}}{d(\cos \theta)^{|m|}} P_{l}(\cos \theta) \\
P_{l}(\cos \theta)=\frac{1}{2^{l} l !} \frac{d^{l}}{d \cos ^{l} \theta} \sin ^{2 l} \theta,
\end{gathered}
$$

with the conversion

$$
\int_{0}^{\pi} d \theta \sin \theta P_{l m}(\cos \theta) P_{l m}(\cos \theta)=C_{l m}^{2}
$$


and

$$
\partial_{\varphi} Y=i m Y, \quad \partial_{\varphi} Y^{*}=-i m Y^{*} .
$$

The even part is given:

$$
\begin{aligned}
& Q^{(+)}=\int_{\Sigma} d r d \theta d \varphi \frac{r^{2} \sin \theta}{1-\frac{2 m}{r}}\left[\Phi_{1}^{1^{*}} \Phi_{1}^{1}+2 r(r-2 m)\left(\Phi_{1}^{2^{*}} \Phi_{1}^{2}+\sin ^{2} \theta \Phi_{1}^{3^{*}} \Phi_{1}^{3}\right)\right. \\
& \left.+2 \sin ^{2} \theta \Phi_{2}^{3 *} \Phi_{2}^{3}+\Phi_{3}^{3^{*}} \Phi_{3}^{3}\right] \\
& =\int_{Z} d r d \theta d \varphi \frac{r^{2} \sin \theta}{1-\frac{2 m}{r}}\left[H^{2} Y^{*} Y+2 r(r-2 m)\left(K^{2} \partial_{\theta} Y^{*} \partial_{\theta} Y\right.\right. \\
& \left.+\frac{K^{2}}{\sin ^{2} \theta} \partial_{\varphi} Y^{*} \partial_{\varphi} Y\right) \\
& +2 G_{2}^{2} \frac{1}{\sin ^{2} \theta}\left[\left(\partial_{\theta}-\cot \theta\right) \partial_{\varphi} Y^{*}\right]\left[\left(\partial_{\theta}-\cot \theta\right) \partial_{\varphi} Y\right] \\
& +\left(G_{1}+G_{2} \partial_{\theta}^{2}\right) Y^{*}\left(G_{1}+G_{2} \partial_{\theta}^{2}\right) Y \\
& +\left(G_{1}+G_{2} \frac{1}{\sin ^{2} \theta} \partial_{\varphi}^{2}+G_{2} \cot \theta \partial_{\theta}\right) Y^{*}\left(G_{1}\right. \\
& \left.\left.+G_{2} \frac{1}{\sin ^{2} \theta} \partial_{\varphi}^{2}+G_{2} \cot \theta \partial_{\theta}\right) Y\right] \\
& =\int_{2 m}^{\infty} d r \frac{r^{2}}{1-\frac{2 m}{r}} H^{2}+\int_{2 m}^{\infty} d r \frac{r^{2}}{1-\frac{2 m}{r}} K^{2} l(l+1) 2 r(r-2 m) \\
& +\int_{\Sigma} d r d \theta d \varphi \frac{r^{2} \sin \theta}{1-\frac{2 m}{r}}\left[2 G_{2}^{2} \partial_{\theta}\left(\frac{1}{\sin \theta} \partial_{\varphi} Y^{*}\right)\right. \\
& \cdot \partial_{\theta}\left(\frac{1}{\sin \theta} \partial_{\varphi} Y\right)+2 G_{1}^{2} Y^{*} Y \\
& +\hat{L} Y^{*} \hat{L} Y G_{2}^{2}+2 G_{1} Y^{*} G_{2} \hat{L} Y-2 G_{2}^{2} \partial_{\theta}^{2} Y^{*} \\
& \left.\cdot\left(\frac{1}{\sin ^{2} \theta} \partial_{\varphi}^{2}+\cot \theta \partial_{\theta}\right) Y\right] \text {. }
\end{aligned}
$$

As a result, we have:

$$
\begin{aligned}
Q^{(+)}= & \int_{2 m}^{\infty} d r \frac{r^{2}}{N^{2}}\left\{H^{2}+l(l+1) K^{2} 2 r(r-2 m)\right. \\
& +2 G_{1}^{2}+l^{2}(l+1)^{2} G_{2}{ }^{2}-2 l(l+1) G_{1} G_{2} \\
& +2 G_{2}^{2} \int d \theta d \varphi \sin \theta\left[\partial_{\theta}\left(\frac{1}{\sin \theta} \partial_{\varphi} Y^{*}\right) \partial_{\theta}\left(\frac{1}{\sin \theta} \partial_{\varphi} Y\right)\right. \\
& \left.\left.-\partial_{\theta}^{2} Y^{*}\left(\frac{1}{\sin \theta} \partial_{\varphi}^{2}+\cot \theta \partial_{\theta}\right) Y\right]\right\}
\end{aligned}
$$

We calculate:

$$
\int d \varphi d \theta \sin \theta\left|\partial_{\theta}\left(\frac{1}{\sin \theta} \partial_{\varphi} Y\right)\right|^{2}
$$




$$
\begin{aligned}
= & \frac{m^{2}}{C_{l m}{ }^{2}} \int_{-1}^{1} d x\left\{\left(1-x^{2}\right)\left[\partial_{x}\left(\left(1-x^{2}\right)^{\frac{m-1}{2}} \partial^{m} P_{l}(x)\right)\right]^{2}\right\} \\
= & \frac{m^{2}}{C_{l m}{ }^{2}} \int_{-1}^{1} d x\left(1-x^{2}\right)\left[-(m-1) x\left(1-x^{2}\right)^{\frac{m-1}{2}-1} \partial^{m} P_{l}(x)\right. \\
& \left.+\left(1-x^{2}\right)^{\frac{m-1}{2}} \partial^{m+1} P_{l}(x)\right]^{2} \\
= & \frac{m^{2}}{C_{l m}{ }^{2}} \int_{-1}^{1} d x\left[(m-1)^{2} x^{2}\left(1-x^{2}\right)^{m-2} \partial^{m} P_{l}(x) \partial^{m} P_{l}(x)\right. \\
& +\left(1-x^{2}\right)^{m} \partial^{m+1} P_{l}(x) \partial^{m+1} P_{l}(x) \\
& \left.-(m-1) x\left(1-x^{2}\right)^{m-1} \partial^{m} P_{l}(x) \partial^{m+1} P_{l}(x)\right] \\
= & {\left[-(m-1)^{2} J_{(m, l)}+(m-1)^{2} K_{(m, l)}\right.} \\
& \left.+J_{(m+1, l)}-2(m-1) H_{(m, l)}\right] \frac{m^{2}}{C_{l m}{ }^{2}}
\end{aligned}
$$

where

$$
\left\{\begin{array}{l}
J_{(m, l)} \equiv \int_{-1}^{1}\left(1-x^{2}\right)^{m-1} \partial^{m} P_{l}(x) \partial^{m} P_{l}(x) d x \\
K_{(m, l)} \equiv \int_{-1}^{1}\left(1-x^{2}\right)^{m-2} \partial^{m} P_{l}(x) \partial^{m} P_{l}(x) d x \\
H_{(m, l)} \equiv \int_{-1}^{1} x\left(1-x^{2}\right)^{m-1} \partial^{m} P_{l}(x) \partial^{m+1} P_{l}(x) d x \\
I_{(m, l)} \equiv-\int_{-1}^{1} x\left(1-x^{2}\right)^{m} \partial^{m} P_{l}(x) \partial^{m+1} P_{l}(x) d x
\end{array}\right.
$$

And:

$$
\begin{aligned}
\int d & \theta d \varphi \sin \theta\left[-\partial_{\theta}^{2} Y^{*}\left(\frac{1}{\sin ^{2} \theta} \partial_{\varphi}^{2}+\cot \theta \partial_{\theta}\right) Y\right] \\
= & \int d \theta d \varphi \sin \theta\left[l(l+1) Y^{*}\left(-\frac{1}{\sin ^{2} \theta} m^{2} Y+\cot \theta \partial_{\theta} Y\right)\right. \\
& \left.+m^{4} \frac{1}{\sin ^{4} \theta} Y^{*} Y+\cot ^{2} \theta \partial_{\theta} Y^{*} \partial \theta Y-2 m^{2} \frac{\cot \theta}{\sin ^{2} \theta} Y^{*} \partial_{\theta} Y\right] \\
= & \left\{l(l+1)\left[-m^{2} J_{(m, l)}+I_{(m, l)}-m C_{l m}{ }^{2}+m J_{(m, l)}\right]\right. \\
& +\left[m^{4} K_{(m, l)}+J_{(m+1, l)}-m^{2} J_{(m, l)}-2 m H_{(m, l)}+m^{2} K_{(m, l)}\right. \\
& -C_{l, m+1}{ }^{2}+m^{2} C_{l m}{ }^{2}-m^{2} J_{(m, l)}-2 m I_{(m, l)} \\
& \left.\left.+2 m^{2} H_{(m, l)}+2 m^{3} J_{(m, l)}-2 m^{3} K_{(m, l)}\right]\right\} \frac{1}{2} .
\end{aligned}
$$

Summing (224) and (226), ones gets 10 :

$$
\begin{aligned}
A_{(m, l)}= & \frac{l(l+1)}{C_{l m}{ }^{2}}\left[J_{m} m(1-m)+I_{m}-C_{l m}{ }^{2}\right] \\
& +\frac{1}{{C_{l m}}^{2}}\left[2 m^{2}(m-1)^{2} K_{m}-C_{l, m+1}{ }^{2}+m^{2} C_{l m}{ }^{2}\right.
\end{aligned}
$$

\footnotetext{
${ }^{10}$ We omit the index $l$.
} 


$$
\begin{aligned}
& +J_{m} m^{2}(m-1)(3-m)+\left(1+m^{2}\right) J_{m+1} \\
& \left.-2 m(m-1)^{2} H_{m}-2 m I_{m}\right] .
\end{aligned}
$$

We calculate explicitly the integral (225) and obtain:

$$
\begin{gathered}
I_{m}=-I_{m}+C_{l m}{ }^{2}-2 m \int_{-1}^{1}\left(1-x^{2}\right)^{m-1} x^{2} \partial^{m} P_{l}(x) \partial^{m} P_{l}(x) d x \\
=-I_{m}+(2 m+1) C_{l m}{ }^{2}-2 m J_{m}
\end{gathered}
$$

thus,

$$
J_{m}=\frac{1}{m} I_{m}+\frac{\left(m+\frac{1}{2}\right)}{m} C_{l m}{ }^{2} .
$$

And

$$
\left(1-x^{2}\right) \partial^{m+1} P_{l}(x)=2 m x \partial^{m} P_{l}(x)-(l+m)(l-m+1) \partial^{m-1} P_{l}(x)
$$

therefore

$$
\begin{aligned}
I_{m} & =-\frac{1}{2 m} C_{l m}{ }^{2}-\frac{(l+m)(l-m+1)}{2 m} \int_{-1}^{1}\left(1-x^{2}\right)^{m} \partial^{m-1} P_{l}(x) \partial^{m+1} P_{l}(x) d x \\
& =-\frac{1}{2 m}\left[C_{l, m+1}{ }^{2}-(l+m)(l-m+1) C_{l, m}{ }^{2}\right. \\
& \left.-(l+m)(l-m+1) 2 m I_{m-1}\right]
\end{aligned}
$$

owing to $C_{l, m+1}{ }^{2}=C_{l, m}{ }^{2}(l+m+1)(l-m)$ e $C_{l, m}{ }^{2}=C_{l, m-1}{ }^{2}(l+m)(l-m+1)$, one has:

$$
\begin{aligned}
I_{m}= & C_{l, m}{ }^{2}+(l+m)(l-m+1) I_{m-1} \\
& =2 C_{l, m}{ }^{2}+(l+m)(l+m-1)(l-m+1)(l-m+2) I_{m-2} \\
& =m^{2} C_{l, m}{ }^{2}+\frac{(l+m) !}{(l-m) !} I_{0} . \\
I_{0}= & -\int_{-1}^{1} x P_{l}(x) \partial P_{l}(x) d x=-I_{0}+\int_{-1}^{1}\left[P_{l}\right]^{2} d x-\left.x P_{l}^{2}(x)\right|_{-1} ^{1} \\
& =\frac{1}{2 l+1}-1 .
\end{aligned}
$$

As a result $I_{m}=(m-l) C_{l, m}{ }^{2}$ e $J_{m}=\frac{2 l+1}{2 m} C_{l, m}{ }^{2}$. Now we have:

$$
\begin{aligned}
J_{m} & =-\int_{-1}^{1} \partial^{m-1} P_{l}(x) \partial\left[\left(1-x^{2}\right)^{m-1} \partial^{m} P_{l}\right] d x \\
& =2(m-1) \int_{-1}^{1} \partial^{m-1} P_{l}(x) \partial^{m} P_{L}(x) x\left(1-x^{2}\right)^{m-2} d x
\end{aligned}
$$




$$
\begin{aligned}
& -\int_{-1}^{1} \partial^{m-1} P_{l}(x) \partial^{m+1} P_{l}(x)\left(1-x^{2}\right)^{m-1} d x \\
= & 2(m-1) H_{m-1}-\int_{-1}^{1} P_{l}(x) \partial^{m+1} P_{l}(x)\left(1-x^{2}\right)^{m-1} d x \\
= & 2(m-1) H_{m-1}-\left[\int_{-1}^{1} \partial^{m-1} P_{l}(x) 2 m x \partial^{m} P_{l}(x)\left(1-x^{2}\right)^{m-2} d x\right. \\
& \left.-(l+m)(l-m+1) \int_{-1}^{1} \partial^{m-1} P_{l}(x) \partial^{m-1} P_{l}(x)\left(1-x^{2}\right)^{m-2} d x\right] \\
= & -2 H_{m-1}+(l+m)(l-m+1) J_{m-1}
\end{aligned}
$$

and then

or

$$
H_{m}=\frac{(l+m+1)(l-m)}{2} J_{m}-\frac{J_{m+1}}{2}
$$

$$
H_{m}=\frac{(2 l+1)(l+m+1)(l-m)}{4 m(m+1)} .
$$

Finally

$$
\begin{aligned}
J_{m}= & \int_{-1}^{1}\left(1-x^{2}\right)^{m+1} \partial^{m} P_{l}(x) \partial^{m} P_{l}(x) d x \\
= & -2 \int_{-1}^{1} x\left(1-x^{2}\right)^{m-1} \partial^{m+1} P_{l}(x) \partial^{m} P_{l}(x) d x \\
& \quad+2(m-1) \int_{-1}^{1} x^{2}\left(1-x^{2}\right)^{m-2} \partial^{m} P_{l}(x) \partial^{m} P_{l}(x) \\
= & -2 H_{m}-2(m-1) J_{m}+2(m-1) K_{m}
\end{aligned}
$$

which leads to:

$$
\begin{aligned}
K_{m}= & \frac{2 m-1}{2(m-1)} J_{m}+\frac{H_{m}}{m-1} \\
& =\frac{2 l+1}{4 m(m-1)}\left[2 m-1+\frac{(l+m+1)(l-m)}{m+1}\right] .
\end{aligned}
$$

Therefore we find:

$$
\begin{aligned}
A_{(l, m)}= & l(l+1)\left[-\frac{2 l+1}{2}(m-1)+m-l-m\right]+\frac{1}{2} m(m-1)(2 m \\
& -1)(2 l+1)+\frac{1}{2} \frac{m(m-1)(2 l+1)(l+m+1)(l-m)}{m+1} \\
& -(l+m+1)(l-m)+m^{2}+\frac{1}{2} m(m-1)(3-m)(2 l+1) \\
& +\frac{1}{2(m+1)}(2 l+1)(l+m+1)(l-m)\left(m^{2}+1\right) \\
& -\frac{1}{2(m+1)}(m-1)^{2}(l+m+1)(l-m)(2 l+1) \\
= & -\frac{1}{2} l(l+1) .
\end{aligned}
$$


Thus one gets:

$$
\begin{aligned}
Q^{(+)} & =\int_{2 m}^{+\infty} \frac{r^{2}}{N^{2}}\left\{H^{2}+l(l+1) K^{2} 2 r(r-2 m)+2 G_{1}^{2}\right. \\
& \left.-2 l(l+1) G_{1} G_{2}+G_{2}^{2}\left[l^{2}(l+1)^{2}-l(l+1)\right]\right\} d r
\end{aligned}
$$

Concerning the odd part, one gets:

$$
\begin{aligned}
Q^{(-)}=\int_{\Sigma} & d r d \theta d \varphi \sin \theta \frac{r^{2}}{N^{2}}\left\{2 r ( r - 2 m ) \left[F_{1}^{2} \frac{1}{\sin ^{2} \theta} \partial_{\varphi} Y^{*} \partial_{\varphi} Y\right.\right. \\
& \left.+F_{1}^{2} \partial_{\theta} Y^{*} \partial_{\theta} Y+2 F_{2}^{2} \frac{1}{\sin ^{2} \theta}\left|\left(\partial_{\theta}-\cot \theta\right) \partial_{\varphi} Y_{l m}\right|^{2}\right] \\
& \left.+\frac{1}{2} F_{2}^{2}\left|\left[l(l+1)+\frac{2}{\sin ^{2} \theta} \partial_{\varphi}^{2}+2 \cot \theta \partial_{\theta}\right] Y\right|^{2}\right\} \\
= & \int_{2 m}^{\infty} d r \frac{r^{2}}{N^{2}}\left\{2 r(r-2 m) F_{1}^{2}(r) l(l+1)+F_{2}^{2}\left[\frac{l^{2}(l+1)^{2}}{2}\right.\right. \\
& +2\left(m ^ { 2 } \int d \omega \left(\left|\partial_{\theta}\left(\frac{1}{\sin \theta} Y\right)\right|^{2}+l(l+1)\left(-\frac{m^{2}}{\sin ^{2} \theta} Y^{*} Y\right.\right.\right. \\
& \left.+\cot \theta \partial_{\theta} Y^{*} Y\right)\left(\frac{m^{4}}{\sin ^{4} \theta} Y^{*} Y+\cot ^{2} \theta \partial_{\theta} Y^{*} \partial_{\theta} Y\right. \\
& \left.\left.\left.\left.\left.-\frac{m^{2}}{\sin ^{2} \theta} \cot ^{2} \theta \partial_{\theta} Y^{*} \partial_{\theta} Y\right)\right)\right)\right]\right\}
\end{aligned}
$$

Using the results from calculating the even part, we obtain:

$$
Q^{(-)}=\int_{2 m}^{\infty} \frac{r^{2}}{N^{2}}\left\{2 r(r-2 m) l(l+1) F_{1}^{2}(r)+F_{2}{ }^{2}\left[\frac{l^{2}(l+1)^{2}}{2}-l(l+1)\right]\right\} d r
$$




\section{Appendix E: Study of eigenequations in the limit of flat space $m=0$}

At the first we study the even part. The eigenequations and the equations of constrains becomes:

$$
\begin{aligned}
& \int\left[\partial_{r}^{2}+\frac{2}{r} \partial_{r}-\frac{l(l+1)}{r^{2}}-\frac{4}{r^{2}}\right] H(r)+\frac{4}{r} l(l+1) K(r) \\
& +\frac{2}{r^{2}}\left(2 G_{1}(r)-l(l+1) G_{2}(r)\right)=-4 \frac{\lambda}{\hbar^{2}} H(r) \\
& {\left[\partial_{r}^{2}+\frac{2}{r} \partial_{r}-\frac{l(l+1)}{r^{2}}+\frac{2}{r^{2}}\right] G_{2}(r)+\frac{4}{r} K(r)=-4 \frac{\lambda}{\hbar^{2}} G_{2}(r)} \\
& {\left[\partial_{r}^{2}+\frac{2}{r} \partial_{r}-\frac{l(l+1)}{r^{2}}-\frac{2}{r^{2}}\right] G_{1}(r)+\frac{2}{r^{2}} G_{2}(r) l(l+1)} \\
& +\frac{2}{r^{2}} H(r)=-4 \frac{\lambda}{\hbar^{2}} G_{1}(r) \\
& {\left[\partial_{r}^{2}+\frac{2}{r} \partial_{r}-\frac{l(l+1)}{r^{2}}-\frac{2}{r^{2}}\right] K(r)+\frac{2}{r^{3}} H(r)-\frac{2}{r^{3}}\left[G_{1}(r)\right.} \\
& \left.\left.+(1-l(l+1)) G_{2}(r)\right)\right]=-4 \frac{\lambda}{\hbar^{2}} K(r)
\end{aligned}
$$

and

$$
\left\{\begin{array}{l}
2 G_{1}(r)-G_{2}(r) l(l+1) H(r)=0 \\
\partial_{r} H(r)+3 \frac{H(r)}{r}-K(r) l(l+1)=0 \\
r^{2} \partial_{r} K(r)+4 r K(r)+G_{2}(r)[1-l(l+1)]=0 .
\end{array}\right.
$$

We show that eigenequations are consistent with equations of constrains. Substituting the first three equations of system (242) into the first equation of (243):

$$
\begin{aligned}
-\frac{4 \lambda}{\hbar^{2}} & {\left[2 G_{1}(r)-l(l+1) G_{2}(r)+H(r)\right]=\left[\partial_{r}^{2}+\frac{2}{r} \partial_{r}-\frac{l(l+1)}{r^{2}}\right] } \\
\cdot & {\left[2 G_{1}(r)-l(l+1) G_{2}(r)+H(r)\right] . }
\end{aligned}
$$

Now we substitute the first and last equations of (242) into the second equation of (243); take into account the relations:

$$
\partial_{r}\left[\frac{2}{r} \partial_{r} H(r)\right]=\frac{2}{r} \partial_{r}\left[\partial_{r} H(r)\right]-\frac{2}{r^{2}} \partial_{r} H(r)
$$




$$
\begin{gathered}
\partial_{r}\left[\frac{2}{r^{2}} \partial_{r} H(r)\right]=\frac{1}{r^{2}} \partial_{r}\left[\partial_{r} H(r)\right]-\frac{2}{r^{3}} \partial_{r} H(r) \\
\frac{1}{r}\left[\partial_{r}^{2} H(r)\right]=\partial_{r}^{2}\left[\frac{1}{r} H(r)\right]+\frac{2}{r^{2}} \partial_{r} H(r)-\frac{2}{r^{3}} H(r) \\
\frac{1}{r}\left[\frac{1}{r} \partial_{r} H(r)\right]=\frac{1}{r} \partial_{r}\left[\frac{1}{r} H(r)\right]+\frac{1}{r^{3}} H(r)
\end{gathered}
$$

and using the third equation of (243), we obtain:

$$
\begin{gathered}
\frac{4 \lambda}{\hbar^{2}}\left[\partial_{r} H(r)+\frac{3 H(r)}{r}-l(l+1) K(r)\right] \\
=\left[\partial_{r}^{2}+\frac{2}{r} \partial_{r}-l(l+1)-\frac{2}{r^{2}}\right] \\
\cdot\left[\partial_{r} H(r)+\frac{3}{r} H(r)-l(l+1) K(r)\right] .
\end{gathered}
$$

Finally, substituting the last three equations of system (242) into the last equation of (243), we have

$$
\begin{gathered}
r^{2} \partial_{r}\left[\partial_{r}^{2} K(r)\right]=\partial_{r}^{2}\left[r^{2} \partial_{r} K(r)\right]-2 \partial_{r} K(r)-4 r \partial_{r}^{2} K(r) \\
r^{2} \partial_{r}\left[\frac{1}{r} \partial_{r} K(r)\right]=\frac{1}{r}\left[r^{2} \partial_{r} K(r)\right]-3 \partial_{r} K(r) \\
r^{2} \partial_{r}\left[\frac{1}{r^{2}} K(r)\right]=\frac{1}{r^{2}}\left[r^{2} \partial_{r} K(r)\right]-\frac{2}{r} K(r) \\
r \partial_{r}^{2} K(r)=\partial_{r}^{2}[r K(r)]-2 \partial_{r} K(r) \\
=\frac{1}{r} \partial_{r}\left[r^{2} \partial_{r} K(r)\right]-2 \partial_{r} K(r) \\
r\left[\frac{1}{r} \partial_{r} K(r)\right]=\frac{1}{r} \partial_{r}[r K(r)]-\frac{1}{r} K(r)
\end{gathered}
$$

and the same for (243), one gets:

$$
\begin{aligned}
-\frac{4 \lambda}{\hbar^{2}} & {\left[r^{2} \partial_{r} K(r)+4 r K(r)+G_{1}(r)+G_{2}(r)(1-l(l+1))\right] } \\
& =\left[\partial_{r}^{2}-\frac{l(l+1)}{r^{2}}\right]\left[r^{2} \partial_{r} K(r)+4 r K(r)\right. \\
& \left.+G_{1}(r)+G_{2}(r)(1-l(l+1))\right] .
\end{aligned}
$$

The equation (244), (245) and (246) show that the conditions of constrain (243) are compatible with the eigenequation (242). Thus, we can solve the eigenequation by using the first equation of (242) together with the eq.(243). As a result, we have an equation for $H(r)$ only:

$$
\left[\partial_{r}^{2}+\frac{6}{r} \partial_{r}+\frac{6-l(l+1)}{r^{2}}\right] H(r)=4 \frac{\lambda}{\hbar^{2}} H(r) .
$$


Introducing the variable $\rho=2 \frac{\sqrt{\lambda}}{\hbar} r$ and indicating " , " as the derivative with respect to new variable, one gets:

$$
H^{\prime \prime}+\frac{6}{\rho} H^{\prime}+\left[1-\frac{(l+3)(l-2)}{\rho^{2}}\right]=0 .
$$

Setting 11

$$
H=\rho^{\alpha} J_{\nu}(\rho)
$$

one gets:

$$
\begin{gathered}
H^{\prime}=\alpha \rho^{\alpha-1} J_{\nu}(\rho)+\rho^{\alpha}{J^{\prime}}_{\nu}(\rho) \\
H^{\prime \prime}=\alpha(\alpha-1) \rho^{\alpha-2} J_{\nu}(\rho)+2 \alpha \rho^{\alpha-1}{J^{\prime}}_{\nu}(\rho)+\rho^{\alpha}{J^{\prime \prime}}_{\nu}(\rho),
\end{gathered}
$$

and substituting it into (248):

$$
\begin{aligned}
& \rho^{\alpha}\left[J_{\nu}{ }_{\nu}(\rho)+\frac{2 \alpha+6}{\rho} J^{\prime}{ }_{\nu}+(1\right. \\
& \left.\left.\quad-\frac{(l+3)(l-2)-6 \alpha-\alpha(\alpha-1)}{\rho^{2}}\right) J_{\nu}(\rho)\right]=0
\end{aligned}
$$

with $2 \alpha+6=1$, so that the above equation is the equation of Bessel function with $\nu^{2}=\left(l+\frac{1}{2}\right)^{2}$. Finally,

$$
H(r)=C r^{-\frac{5}{2}} J_{l+\frac{1}{2}}\left(2 \frac{\sqrt{\lambda}}{\hbar} r\right) .
$$

From the second equation of (243), one finds:

$$
\begin{aligned}
K(r)= & \frac{c}{l p}\left[\frac{r^{-7 / 2}}{2} J_{l+\frac{1}{2}}\left(2 \frac{\sqrt{\lambda}}{\hbar} r\right)\right. \\
& \left.-\frac{2 \sqrt{\lambda}}{\hbar} \frac{1}{r^{5 / 2}} J^{\prime}{ }_{l+\frac{1}{2}} \frac{2 \sqrt{\lambda} r}{\hbar}\right] .
\end{aligned}
$$

Recalling the relation for the Bessel function

$$
\frac{d}{d z}\left(z^{-\nu} J_{\nu}(z)\right)=-z^{-\nu} J_{\nu+1}(z),
$$

we have

$$
J^{\prime}{ }_{l+\frac{1}{2}}\left(2 \frac{\sqrt{\lambda}}{\hbar} r\right)=\frac{\hbar}{2 \sqrt{\lambda}} r\left(l+\frac{1}{2}\right) J_{l+\frac{1}{2}}\left(2 \frac{\sqrt{\lambda}}{\hbar} r\right)-J_{l+\frac{3}{2}}\left(2 \frac{\sqrt{\lambda}}{\hbar} r\right),
$$

\footnotetext{
${ }^{11} J_{\nu}$ are the Bessel functions.
} 
thus

$$
K(r)=\frac{c}{l(l+1)}\left[\frac{l+1}{r^{7 / 2}} J_{l+\frac{1}{2}}\left(2 \frac{\sqrt{\lambda} r}{\hbar}\right)-\frac{2 \sqrt{\lambda}}{\hbar} \frac{1}{r^{5 / 2}} J_{l+\frac{3}{2}}\left(2 \frac{\sqrt{\lambda} r}{\hbar}\right)\right] .
$$

From the first equation of (243) one finds:

$$
2 G_{1}(r)-G_{2}(r) l(l+1)+c r^{-5 / 2} J_{l+\frac{1}{2}}\left(2 \frac{\sqrt{\lambda} r}{\hbar}\right)=0 .
$$

From the last, instead, one find:

$$
\begin{aligned}
G_{1}(r) & +G_{2}(r)[1-l(l+1)]=-\frac{4 c}{l(l+1)}\left\{\frac{l+1}{r^{5 / 2}} J_{l+\frac{1}{2}}\left(2 \frac{\sqrt{\lambda}}{\hbar}\right)\right. \\
& -2 \frac{\sqrt{\lambda}}{\hbar r^{3 / 2} J_{l+\frac{3}{2}}}\left(2 \frac{\sqrt{\lambda} r}{\hbar}\right)-\frac{c}{l(l+1)}\left[-\frac{7}{2} \frac{l+1}{r^{5 / 2}} J_{l+\frac{1}{2}}\left(2 \frac{\sqrt{\lambda} r}{\hbar}\right)\right. \\
& +\frac{l+1}{r^{3 / 2}} 2 \frac{\sqrt{\lambda}}{\hbar} J^{\prime}{ }_{l+\frac{1}{2}}\left(2 \frac{\sqrt{\lambda} r}{\hbar}\right)+\frac{5}{2} \frac{2 \sqrt{\lambda}}{\hbar r^{3 / 2}}\left(2 \frac{\sqrt{\lambda} r}{\hbar}\right) \\
& \left.\left.-\frac{4}{r^{1 / 2}} \frac{\lambda}{\hbar^{2}} J^{\prime}{ }_{l+\frac{3}{2}}(2 \sqrt{\lambda} r)\right]\right\} \\
= & -\frac{c}{l\left(l+\frac{1}{2}\right)}\left[\frac{(l+1)^{2}}{r^{5 / 2}} J_{l+\frac{1}{2}}\left(2 \frac{\sqrt{\lambda} r}{\hbar}\right)-\frac{2 l+3}{r^{3 / 2}} 2 \frac{\sqrt{\lambda}}{\hbar} J_{l+\frac{3}{2}}\left(2 \frac{\sqrt{\lambda} r}{\hbar}\right)\right. \\
& \left.+\frac{4}{r^{1 / 2}} \frac{\lambda}{\hbar^{2}} J_{l+\frac{5}{2}}\left(2 \frac{\sqrt{\lambda} r}{\hbar}\right)\right] .
\end{aligned}
$$

At this point, we can adopt approximation $\hbar \rightarrow 0$, which is equivalent to the limit of $J_{\nu+\frac{1}{2}}(z)$ for $z \rightarrow+\infty$,

$$
J_{\nu+\frac{1}{2}}(z) \sim \sqrt{\frac{2}{\pi}} \frac{1}{\sqrt{z}} \cos \left[z-(\nu+1) \frac{\pi}{2}\right]
$$

and one has:

$$
\begin{gathered}
H(r) \sim C \sqrt{\frac{1}{\pi} \hbar \sqrt{\frac{2}{\lambda}}} \frac{1}{r^{3}} \cos \left[\frac{\sqrt{\lambda} r}{\hbar}-l(l+1) \frac{\pi}{2}\right] \\
K(r) \sim \frac{C}{l(l+1)} \sqrt{\frac{\hbar}{\pi} \sqrt{\frac{2}{\lambda}}} 2 \frac{\sqrt{\lambda} r}{\hbar} \cos \left[2 \frac{\sqrt{\lambda} r}{\hbar}-(l+2) \frac{\pi}{2}\right] \\
=\frac{1}{l(l+1)} 2 \sqrt{\lambda} c \sqrt{\frac{1}{\pi} \sqrt{\frac{2}{\lambda}}} \sin \left[2 \frac{\sqrt{\lambda} r}{\hbar}-l(l+1) \frac{\pi}{2}\right]
\end{gathered}
$$




$$
\begin{aligned}
& 2 G_{1}(r)-G_{2}(r) l(l+1) \sim-c \sqrt{\frac{\hbar}{\pi} \sqrt{\frac{2}{\lambda}}} \frac{1}{r^{3}} \cos \left[2 \frac{\sqrt{\lambda} r}{\hbar}-(l+1) \frac{\pi}{2}\right] \\
& G_{1}(r)+G_{2}(r)[1-l(l+1)]
\end{aligned}
$$

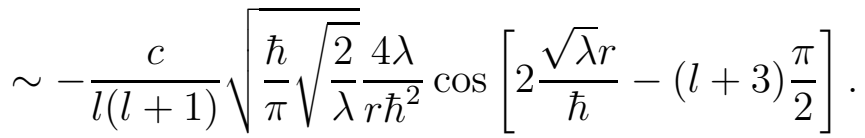

From the last two equations, one finds:

$$
G_{1}(r) \sim \frac{l(l+1)}{2} G_{2}(r)
$$

con

$$
\begin{aligned}
G_{2}(r) \sim & \frac{1}{1-\frac{l(l+1)}{2}} \frac{c}{l(l+1)} \sqrt{\frac{\hbar}{\pi} \sqrt{\frac{2}{\lambda}}} \\
& \cdot \frac{4 \lambda}{r \hbar^{2}} \cos \left[2 \frac{\sqrt{\lambda} r}{\hbar}-(l+1) \frac{\pi}{2}\right] .
\end{aligned}
$$

It is clear then that the (101) coincide with these solutions in the limit $m \rightarrow 0$ if $\alpha(l)=i(l+1) \frac{\pi}{2}$.

Now we study the odd part. In this case, the equation becomes:

$$
\left\{\begin{array}{c}
{\left[\partial_{r}^{2}+\frac{6}{r} \partial_{r}-\frac{l(l+1)}{r^{2}}+\frac{6}{r^{2}}\right] F_{1}(r)=-4 \lambda F_{1}(r)} \\
{\left[\partial_{r}^{2}+\frac{2}{r} \partial_{r}-\frac{l(l+1)}{r^{2}}+\frac{2}{r^{2}}\right] F_{2}(r)-\frac{4}{r} F_{1}(r)} \\
=-4 \lambda F_{2}(r) \\
F_{2}(r)\left(1-\frac{l(l+1)}{2}\right)=\left(r^{2} \partial_{r}+4 r\right) F_{1}(r) .
\end{array}\right.
$$

Again the conditions of constrain are compatible with the eigenequation. In fact one finds:

$$
\begin{aligned}
-4 \lambda & {\left[\left(r^{2} \partial_{r}+4 r\right) F_{1}(r)-\left(1-\frac{l(l+1)}{2}\right) F_{2}(r)\right] } \\
& =\left(r^{2} \partial_{r}+4 r\right)\left[\partial_{r}^{2}+\frac{6}{r} \partial_{r}-\frac{l(l+1)}{r^{2}}+\frac{6}{r^{2}}\right] F_{1}(r) \\
& -\left[1-\frac{l(l+1)}{2}\right]\left[\partial_{r}^{2}+\frac{2}{r} \partial_{r}-\frac{l(l+1)}{r^{2}}+\frac{2}{r^{2}}\right] F_{2}(r) \\
& +\left[1-\frac{l(l+1)}{2}\right] \frac{4}{r} F_{1}(r) .
\end{aligned}
$$


Based on:

$$
\begin{gathered}
r^{2} \partial_{r}\left[\partial_{r}^{2} F_{1}(r)\right]=\partial_{r}^{2}\left[r^{2} \partial_{r} F_{1}(r)\right]-2 \partial_{r} F_{1}(r)-4 r \partial_{r}^{2} F_{1}(r) \\
r^{2} \partial_{r}\left[\frac{1}{r} \partial_{r} F_{1}(r)\right]=\frac{1}{r} \partial_{r}\left[r^{2} \partial_{r} F_{1}(r)\right]-3 \partial_{r} F_{1}(r) \\
r^{2} \partial_{r}\left[\frac{1}{r^{2}} F_{1}(r)\right]=\frac{1}{r^{2}} \partial_{r}\left[r^{2} \partial_{r} F_{1}(r)\right]-\frac{2}{r} F_{1}(r) \\
r \partial_{r}^{2} F_{1}(r)=\partial_{r}^{2}\left[r F_{1}(r)\right]-2 \partial_{r} F_{1}(r) \\
r\left[\frac{1}{r} \partial_{r} F_{1}(r)\right]=\frac{1}{r} \partial_{r}\left[r F_{1}(r)\right]-\frac{1}{r} F_{1}
\end{gathered}
$$

one finds

$$
\begin{aligned}
-4 \lambda & {\left[\left(r^{2} \partial_{r}+4 r\right) F_{1}(r)-\left(1-\frac{l(l+1)}{2}\right) F_{2}(r)\right] } \\
= & {\left[\partial_{r}^{2}+\frac{2}{r} \partial_{r}-\frac{l(l+1)}{r^{2}}+\frac{2}{r^{2}}\right] } \\
& \cdot\left\{\left[r^{2} \partial_{r}+4 r\right] F_{1}(r)-\left[1-\frac{l(l+1)}{2}\right] F_{2}(r)\right\}
\end{aligned}
$$

which show the compatibility of constrain. From the first equation of (263), one gets:

$$
F_{1}(r)=\frac{c}{r^{5 / 2}} J_{l+\frac{1}{2}}\left(2 \frac{\sqrt{\lambda} r}{\hbar}\right)
$$

As a consequence:

$$
\begin{aligned}
(1- & \left.\frac{l(l+1)}{2}\right) F_{2}(r)=c\left[-\frac{5}{2} \frac{1}{r^{3 / 2}} J_{l+\frac{1}{2}}\left(2 \frac{\sqrt{\lambda} r}{\hbar}\right)\right. \\
& \left.+\frac{2 \sqrt{\lambda}}{r^{1 / 2} \hbar} J^{\prime}{ }_{l+\frac{1}{2}}\left(2 \frac{\sqrt{\lambda} r}{\hbar}\right)+\frac{4}{r^{3 / 2}} J_{l+\frac{1}{2}}\left(2 \frac{\sqrt{\lambda} r}{\hbar}\right)\right] \\
= & c\left[\frac{l+2}{r^{3 / 2}} J_{l+\frac{1}{2}}\left(2 \frac{\sqrt{\lambda} r}{\hbar}\right)-\frac{2 \sqrt{\lambda} r^{1 / 2}}{\hbar} J_{l+\frac{3}{2}}\left(2 \frac{\sqrt{\lambda} r}{\hbar}\right)\right] .
\end{aligned}
$$

In the limit $\hbar \rightarrow 0$, one finds:

$$
\begin{aligned}
& F_{1}(r) \sim c \sqrt{\frac{\hbar}{\pi} \sqrt{\frac{2}{\lambda}}} \frac{1}{r^{3}} \cos \left(2 \frac{\sqrt{\lambda} r}{\hbar}-l(l+1) \frac{\pi}{2}\right) \\
& \left(1-\frac{l(l+1)}{2}\right) F_{2}(r) \sim c \sqrt{\frac{\hbar}{\pi} \sqrt{\frac{2}{\lambda}}} \frac{1}{2} \frac{2 \sqrt{\lambda}}{\hbar} \cos \left(2 \frac{\sqrt{\lambda} r}{\hbar}-(l+2) \frac{\pi}{2}\right)
\end{aligned}
$$

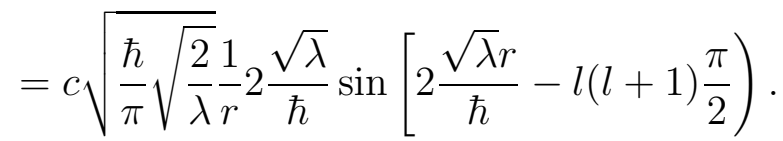

Again $\alpha(l)=-i(l+1)$. 


\section{References}

[1] S. Cacciatori, G. Preparata, S. Rovelli, I. Spagnolatti and S.-S. Xue, On the ground state of quantum gravity, Phys. Lett. B427 (1998) 254.

[2] G. Preparata, Nuovo Cimento A96 (1986) 366.

[3] K. Schwarzschild, Sitzber. Deut. Akad. Wiss. Berlin, Kl, Math.-Phys. Tech. (1916) 189.

[4] T. Regge and J.A. Wheeler, Phys. Rev. 108 (1957) 1063.

[5] D.J. Gross, M.J. Perry and L.G. Yaffe, Phys. Rev. D25 (1982) 330.

[6] P.A.M. Dirac, Phys. Rev. 114 (1959) 924.

[7] R. Arnowitt, S. Deser and C.W. Misner, Phys. Rev. 116 (1959) 1322.

[8] B.S. DeWitt, Phys. Rev. 160 (1967) 1113.

[9] J.A. Wheeler, Geometrodynamics (Accademic Press, Società Italiana di Fisica, 1962)

[10] R. Schoen, S.T. Yau, Phys. Rev. Lett. 42 (1979) 547; Commun. Math. Phys. 65 (1979) 45; Commun. Math. Phys. 79 (1981) 231.

[11] G.W. Gibbons, Malcolm J. Perry, Phys. Rev. D 22, 313 (1980)

[12] H. Politzer, Phys. Rev. Lett. 30 (1973) 1346;

D. Gross, F. Wilczek, Phys. Rev. Lett. 30 (1973) 1342.

[13] G. Preparata and S.-S. Xue, Phys. Lett. B264 (1991) 35; ibid. B335 (1994) 192; Nucl. Phys. B26 (Proc. Suppl.)(1992) 501; ibid. B30 (Proc. Suppl.) (1993) 647.

[14] C.W. Misner, K. Thorne, J.A. Wheeler, Gravitation (Freeman, San Francisco, CA, 1973) 